\title{
Hierarchic Finite Element Bases on Unstructured Tetrahedral Meshes *
}

\author{
Mark Ainsworth ${ }^{\dagger} \quad$ Joe Coyle ${ }^{\ddagger}$
}

May 14, 2002

\begin{abstract}
The problem of constructing hierarchic bases for finite element discretisation of the spaces $H^{1}, \boldsymbol{H}(\mathbf{c u r l}), \boldsymbol{H}(\operatorname{div})$ and $L_{2}$ on tetrahedral elements is addressed. A simple and efficient approach to ensuring conformity of the approximations across element interfaces is described. Hierarchic bases of arbitrary polynomial order are presented. It is shown how these may be used to construct finite element approximations of arbitrary, non-uniform, local order approximation on unstructured meshes of curvilinear tetrahedral elements.
\end{abstract}

AMS subject classifications $78-08,65 \mathrm{~N} 30$.

Key words Hierarchic finite element bases. Conforming finite element spaces.

\section{Introduction}

The variational formulation of partial differential equations arising in science and engineering generally involves one or more of the function spaces $H^{1}(\Omega)$, $\boldsymbol{H}(\operatorname{curl} ; \Omega), \boldsymbol{H}(\operatorname{div} ; \Omega)$ and $L_{2}(\Omega)$. The spaces differ in the smoothness properties required of their members. The space $L_{2}(\Omega)$ involves the comparatively modest requirement that its members should be square integrable, while the space $H^{1}(\Omega)$ in addition requires all components of the gradients to be square integrable. The spaces $\boldsymbol{H}(\mathbf{c u r l} ; \Omega)$ and $\boldsymbol{H}(\mathbf{d i v} ; \Omega)$ consist of vector-valued functions and fall in between $L_{2}$ and $H^{1}$ in the smoothness requirements by asking that certain combinations of first derivatives are square integrable:

$$
\boldsymbol{H}(\operatorname{curl} ; \Omega)=\left\{\boldsymbol{v} \in \boldsymbol{L}_{2}(\Omega): \operatorname{curl} \boldsymbol{v} \in \boldsymbol{L}_{2}(\Omega)\right\}
$$

and

$$
\boldsymbol{H}(\operatorname{div} ; \Omega)=\left\{\boldsymbol{v} \in \boldsymbol{L}_{2}(\Omega): \operatorname{div} \boldsymbol{v} \in L_{2}(\Omega)\right\} .
$$

${ }^{*}$ The support of this work by the Engineering and Physical Sciences Research Council of Great Britain under grant GR/M59426 is gratefully acknowledged.

${ }^{\dagger}$ Mathematics Department, Strathclyde University, 26 Richmond St., Glasgow G1 1XH, Scotland. M.Ainsworth@strath.ac.uk

${ }^{\ddagger}$ Mathematics Department, Strathclyde University, 26 Richmond St., Glasgow G1 1XH, Scotland. ra.jcoy@maths.strath.ac.uk 
The finite element method is frequently used to approximate the solutions of boundary value problems. A family of finite element subspaces should respect the smoothness requirements of the underlying function space associated with the problem to be approximated. The most commonly occurring case involves the space $H^{1}(\Omega)$ where the smoothness requirements translate into the fact that a finite element subspace must consist of functions that are continuous across inter-element boundaries. At the opposite extreme the space $L_{2}(\Omega)$, which frequently occurs in the setting of mixed finite element schemes, imposes no inter-element continuity requirements on the finite element subspace.

The spaces $\boldsymbol{H}(\boldsymbol{c u r l} ; \Omega)$ and $\boldsymbol{H}(\mathbf{d i v} ; \Omega)$, although less common, are nevertheless of considerable importance in application areas such as electromagnetism, elasticity and fluid mechanics. The inter-element continuity requirements or conformity conditions are more complicated for these spaces. The space $\boldsymbol{H}(\operatorname{div} ; \Omega)$ requires the normal component of the finite element function to be continuous across an interface, while the space $\boldsymbol{H}(\mathbf{c u r l} ; \Omega)$ requires that certain tangential components of the finite element approximation should be continuous. These conditions are weaker than those associated with the space $H^{1}(\Omega)$, but their realisation can prove problematic nevertheless. One possible alternative consists of using the more familiar vector-valued $\boldsymbol{H}^{1}(\Omega)$-conforming finite element space in place of an $\boldsymbol{H}(\mathbf{d i v} ; \Omega)$ or $\boldsymbol{H}(\mathbf{c u r l} ; \Omega)$-conforming approximation. Unfortunately, this means that the approximation is overly constrained and opens up the possibility that the finite element scheme may not actually converge to the solution of the problem in hand.

The construction of low order $\boldsymbol{H}(\mathbf{d i v})$ and $\boldsymbol{H}(\mathbf{c u r l})$ conforming approximation may be found in textbooks $[7,9,18,31]$. However, interest in the use of higher order finite element schemes, such as $p, h p$ or spectral element methods, has become increasingly widespread. These methods typically involve the use of elements of variable, non-uniform order approximation on unstructured meshes [30, 32]. The use of a higher order space can lead to complications with enforcing appropriate conformity properties of globally defined basis functions across element interfaces, particularly if elements of non-uniform local order are employed.

The treatment of the $L_{2}$ and $H^{1}$-conforming finite element spaces is well documented in the literature. For example, sets of hierarchic basis functions of arbitrary order on tetrahedral elements may be found in the books of Szabo and Babuska [32], and Karniadakis and Sherwin [21]. These spaces are also discussed in the present work, partly in the interests of completeness and partly in order to set the work in context. However, our main reason for revisiting these simpler spaces is to highlight in a familiar setting some of the difficulties which, though not always apparent, are the crux of the problem of constructing conforming spaces on unstructured meshes.

A survey of (low order) finite element schemes geared towards $h$-version discretisation of $\boldsymbol{H}(\mathbf{d i v})$, and to a lesser extent $\boldsymbol{H}(\mathbf{c u r l})$, may be found in the book of Brezzi and Fortin [9]. The basic framework for finite element spaces of arbitrary order on tetrahedral elements was described by Nedelec [28, 29]. The work of Hiptmair [17] is remarkable in that it encompasses a unified treatment of finite element discretisation of both spaces $\boldsymbol{H}(\mathbf{c u r l})$ and $\boldsymbol{H}(\mathbf{d i v})$ within the 
framework of differential forms.

The lowest order finite element schemes for the discretisation of $\boldsymbol{H}$ (curl) are often referred to as edge elements [6, 26, 33] or Whitney forms [36]. The search for a convenient set of basis functions for higher order approximation has attracted a great deal of attention. Several variants of second order basis functions have been described in [10, 14, 19, 23], while third order elements have been considered in $[8,20,24,27]$. A hierarchic set of basis functions up to third order has been presented by Webb and Forghani [35] while Anderson and Volakis [5] target efforts on the construction of higher order functions based on the Whitney forms. The construction of elements of higher than third order is considered by Graglia et.al. [16] where a nodal basis is described, and Webb [34] where basis functions of arbitrary order are presented based on the degrees of freedom outlined in [29]. Demkowicz and Vardapetyan [13] derived families of non-uniform arbitrary order spaces for $\boldsymbol{H}(\mathbf{c u r l})$ and $\boldsymbol{H}(\mathbf{d i v})$ and showed [13] that the vital commuting diagram property [9] is preserved.

Despite the intensive search for hierarchic bases for arbitrary order finite element discretisation of the spaces $\boldsymbol{H}(\mathbf{c u r l})$ and $\boldsymbol{H}(\mathbf{d i v})$, a number of problems remain with the realisation. Most, if not all, of the existing works are limited to low order elements of uniform degree and focus on describing the basis functions on a reference tetrahedron devoting little attention to the problem of ensuring that the associated global basis functions satisfy the global conformity conditions. However, the work of Demkowicz et. al. [12, 13], deals with approximation of arbitrary order. The problem of deriving bases for non-uniform order approximation of $\boldsymbol{H}$ (curl) and $\boldsymbol{H}$ (div) in two dimensions on hybrid meshes of triangular and quadrilateral elements was studied in [1].

The purpose of the current work is to obtain families of hierarchic basis functions of arbitrary polynomial order and to describe the process whereby these functions may be used to construct arbitrary, non-uniform local polynomial order finite element subspaces for $H^{1}(\Omega), \boldsymbol{H}(\mathbf{c u r l} ; \Omega), \boldsymbol{H}(\mathbf{d i v} ; \Omega)$ and $L_{2}(\Omega)$ on general unstructured meshes of possibly curvilinear tetrahedra. Thus, the current work presents a reasonably complete solution to the problem of developing bases on tetrahedral meshes, although some important issues such as conditioning of the matrices are not addressed.

The remainder of the manuscript is organised as follows. Firstly, the problem of enforcing continuity across element boundaries on an unstructured mesh of tetrahedral elements is discussed. Fully hierarchic bases of arbitrary uniform polynomial order are then exhibited for the four spaces of interest. Finally, the generalisation to non-uniform local order of approximation is discussed along with the details of the implementation.

\section{The Problem of Enforcing Conformity}

Traditionally, finite elements as described, for example by Ciarlet [11], are constructed with degrees of freedom identified with the values of a function, or appropriate derivatives, at nodes located on the element. However, when higher or non-uniform local orders are required, as is the case for the $h p$-version finite 


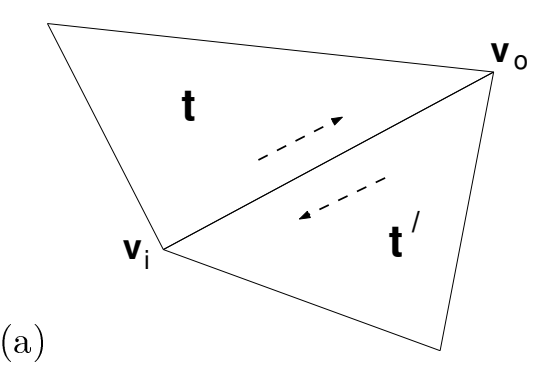

(a)

Figure 1: (a) Triangular elements $\boldsymbol{t}$ and $\boldsymbol{t}^{\prime}$ sharing a common edge $\boldsymbol{e}$ connecting the vertices $\boldsymbol{v}_{o}$ and $\boldsymbol{v}_{i}$. The local orientation of the edge with each element is indicated by the dashed arrows. (b) Local cubic edge basis functions on each element. The mismatch in the local orientation of the edges means that the values of the local degrees of freedom corresponding to the edge function must be appropriately constrained to ensure conformity.

element method, it is advantageous to use degrees of freedom corresponding to hierarchic basis functions [4, 32]. Unfortunately, hierarchic bases create problems with enforcing conformity of the approximation across element interfaces that do not arise with the traditional nodal bases. Here, we illustrate the types of problem that arise in the setting of the scalar $H^{1}(\Omega)$ space. Similar, but more acute, difficulties are encountered in the finite element discretisation of the spaces $\boldsymbol{H}$ (curl) and $\boldsymbol{H}(\mathbf{d i v})$.

\subsection{Enforcing $H^{1}(\Omega)$-Conformity in Two Dimensions}

Suppose that the polygonal domain $\Omega$ is partitioned into triangles so that the non-empty intersection of distinct elements is a single common edge or vertex of both elements. Consider the situation where the order of approximation is chosen to be cubic, $p=3$, and let $u$ be a function from the finite element space. The restriction of $u$ to an inter-element edge $\boldsymbol{e}$ shared by elements $\boldsymbol{t}$ and $\boldsymbol{t}^{\prime}$ (see Figure 1) will be a cubic function of the parametrisation $\xi \in[-1,1]$ used on the edge.

Traditionally, the function would be described by values at four nodes on the edge and these values would correspond to values of local degrees of freedom on both elements $\boldsymbol{t}$ and $\boldsymbol{t}^{\prime}$. The situation is rather different when a hierarchic basis is employed. For instance, a natural choice of hierarchic basis functions, when restricted to the edge, would give the functions

$$
\frac{1}{2}(1-\xi) ; \quad \frac{1}{2}(1+\xi) ; \quad 1-\xi^{2} ; \quad \xi\left(1-\xi^{2}\right) .
$$

If the local orientation of the edges on the reference element is chosen so that the boundary is traversed in an anti-clockwise sense, then the local orientations of the common edge are mismatched, as shown in Figure 1(a). As a consequence, 
the local parametrisation of the edges in each element, denoted by $\xi_{\boldsymbol{t}}$ and $\xi_{\boldsymbol{t}^{\prime}}$, satisfies $\xi_{t}=-\xi_{t^{\prime}}$. This creates difficulties in enforcing continuity across the edge. For instance, suppose $u$ vanishes at the endpoints of the edge $\boldsymbol{e}$ so that the first two functions in (1) are redundant. Relative to the basis functions on element $\boldsymbol{t}$, the restriction of $u$ to the edge is given by

$$
\left.u\right|_{e}=c_{0}\left(1-\xi_{\boldsymbol{t}}^{2}\right)+c_{1} \xi_{\boldsymbol{t}}\left(1-\xi_{\boldsymbol{t}}^{2}\right),
$$

while relative to element $\boldsymbol{t}^{\prime}$,

$$
\left.u\right|_{\boldsymbol{e}}=c_{0}^{\prime}\left(1-\xi_{\boldsymbol{t}^{\prime}}^{2}\right)+c_{1}^{\prime} \xi_{\boldsymbol{t}^{\prime}}\left(1-\xi_{\boldsymbol{t}^{\prime}}^{2}\right) .
$$

In order to obtain a conforming approximation, the values of these different representations must agree on the interface. Inserting $\xi_{e^{\prime}}=-\xi_{e}$ into the latter equation and comparing coefficients leads to the conclusion

$$
\left.\begin{array}{rrr}
c_{0} & = & c_{0}^{\prime} \\
c_{1} & = & -c_{1}^{\prime}
\end{array}\right\} .
$$

The reason behind the sign difference is illustrated in Figure 1(b) where the restrictions of the local cubic edge basis function on the edge are shown. It is observed that the cubic modes have differing signs due to the mismatch in the local orientations of the edge.

The need to resolve such sign conflicts [4] is typical when hierarchic basis functions are used, but does not arise for nodal basis functions. While it is a relatively trivial matter to apply these constraints by incorporating a sign change in the local-global (connectivity) mapping for the element [3], the situation in three dimensions is not so simple.

\subsection{Enforcing $H^{1}(\Omega)$-Conformity in Three Dimensions}

The basic difficulty in enforcing $H^{1}(\Omega)$-conformity in three dimensions first manifests itself in the simple case where fourth order, $p=4$, elements are used and the domain $\Omega$ consists of only two elements. Such a situation is shown in Figure 2, where the tetrahedra, $\boldsymbol{t}$ and $\boldsymbol{t}^{\prime}$, share a common face $\boldsymbol{f}$ defined by nodes with global numbers 2,3 and 4 .

If the boundary value problem is subject to homogeneous boundary conditions, then the finite element functions must vanish on the boundary of the domain $\Omega$. Let $\lambda_{2}, \lambda_{3}$ and $\lambda_{4}$ denote the usual barycentric, or area, coordinates. A cubic finite element function that vanishes on the boundary of the face $\boldsymbol{f}$, but is non-zero on the face itself, must be a multiple of the polynomial $\beta_{\boldsymbol{f}}$ given by $\lambda_{2} \lambda_{3} \lambda_{4}$. Consequently, on the face $\boldsymbol{f}$, the basis for the third order space must reduce to a multiple of the function $\beta_{\boldsymbol{f}}$.

If the order of approximation is increased to fourth order, $p=4$, and a hierarchic basis is required, then the basis functions on the face must, in addition to the function $\beta_{\boldsymbol{f}}$, include two further functions of the form $\beta_{\boldsymbol{f}} w$, where $w$ is an affine function. The natural choice for the two additional functions on element 


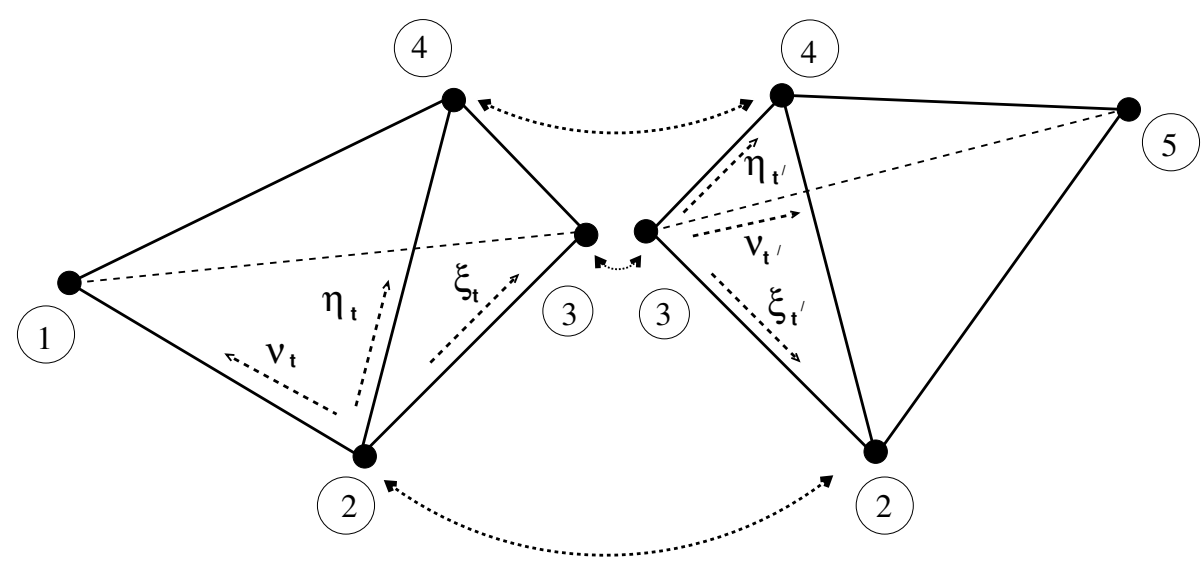

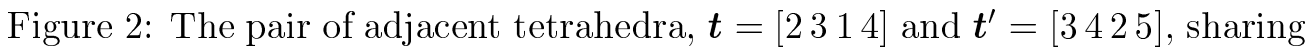
a common face $\boldsymbol{f}$ defined by the vertices with global numbers 2, 3 and 4 . The natural coordinates for each element are indicated by the dashed arrows.

$\boldsymbol{t}$ corresponds to taking $w$ to be $\xi_{\boldsymbol{t}}$ and $\eta_{\boldsymbol{t}}$, as shown in Figure 2, giving the following local face basis functions on element $\boldsymbol{t}$,

$$
\beta_{\boldsymbol{f}} ; \quad \beta_{\boldsymbol{f}} \xi_{\boldsymbol{t}} ; \quad \beta_{\boldsymbol{f}} \eta_{\boldsymbol{t}} .
$$

By the same token, the local face basis functions on $\boldsymbol{t}^{\prime}$ are given by

$$
\beta_{\boldsymbol{f}} ; \quad \beta_{\boldsymbol{f}} \xi_{\boldsymbol{t}^{\prime}} ; \quad \beta_{\boldsymbol{f}} \eta_{\boldsymbol{t}^{\prime}} .
$$

A quartic finite element function $u$ that is non-zero on the face $\boldsymbol{f}$, relative to the basis functions for element $\boldsymbol{t}$, has the form

$$
\left.u\right|_{\boldsymbol{f}}=\beta_{\boldsymbol{f}}\left(c_{0}+c_{1} \xi_{\boldsymbol{t}}+c_{2} \eta_{\boldsymbol{t}}\right)
$$

whilst relative to element $\boldsymbol{t}^{\prime}$, the function has the form

$$
\left.u\right|_{\boldsymbol{f}}=\beta_{\boldsymbol{f}}\left(c_{0}^{\prime}+c_{1}^{\prime} \xi_{\boldsymbol{t}^{\prime}}+c_{2}^{\prime} \eta_{\boldsymbol{t}^{\prime}}\right) .
$$

The relationship between the coefficients needed to ensure agreement on the face can be deduced by first observing that

$$
\xi_{\boldsymbol{t}^{\prime}}=\lambda_{2} ; \quad \eta_{\boldsymbol{t}^{\prime}}=\lambda_{4}
$$

or, noting that $\lambda_{2}+\lambda_{3}+\lambda_{4}=1$ on $\boldsymbol{f}$,

$$
\xi_{\boldsymbol{t}^{\prime}}=1-\lambda_{3}-\lambda_{4} ; \quad \eta_{\boldsymbol{t}^{\prime}}=\lambda_{4} .
$$

Similarly,

$$
\xi_{\boldsymbol{t}}=\lambda_{3} ; \quad \eta_{\boldsymbol{t}}=\lambda_{4},
$$

and so, inserting these into equations (3)-(4) and equating coefficients, we obtain

$$
\left.\begin{array}{l}
c_{0}=c_{0}^{\prime}+c_{1}^{\prime} \\
c_{1}=-c_{1}^{\prime} \\
c_{2}=-c_{1}^{\prime}+c_{2}^{\prime}
\end{array}\right\} .
$$




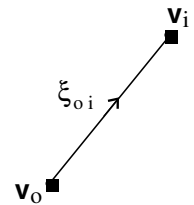

(a) Edge orientation



(b) Face orientation



(c) Tetrahedral orientation

Figure 3: Intrinsic orientations of entities based on the global numbering of the vertices defining the entity. Here, it is assumed that the global numbering satisfies $o<i<j<k$. In each case, the vertex $\boldsymbol{v}_{o}$ with the lowest global numbering is singled out as the preferred vertex.

By way of contrast to the two dimensional case, the application of these constraints is no longer simply a matter of resolving sign conflicts. Indeed, the non-local nature of the constraints makes even the task of identifying the global degrees of freedom non-trivial.

The source of the difficulty may be traced to the freedom in the choice of the two functions needed to augment the basis function already present in the cubic space, to form a basis for the fourth order space. This leads to a set of face basis functions that do not remain invariant under a cycling of the numbering of the vertices on the face. This, in turn, is responsible for the more complicated, nonlocal constraints (5). It is not difficult to convince oneself of the impossibility of constructing hierarchic basis while maintaining rotational invariance. In contrast, a natural choice of nodal basis is automatically rotationally invariant and non-local constraints do not arise. The subject of rotational invariance is discussed further by Webb [34].

\subsection{Circumventing Sign Conflicts}

The foregoing discussion shows that a hierarchic, conforming basis in two dimensions can be realised purely by resolving sign conflicts. However, this notion does not generalise to the three dimensional case due to the loss of rotational invariance in the face basis functions. Faced with this impasse, we shall revisit the two dimensional case in search of an alternative concept that is more readily extended to the three dimensional setting.

Akin [4] shows that, in two dimensions, it is possible to avoid sign conflicts altogether by employing a consistent orientation of edges. An edge $\boldsymbol{e}$ connecting the global vertices $\boldsymbol{v}_{o}$ and $\boldsymbol{v}_{i}$, is assigned a unique, global orientation by choosing, for instance, the tangent vector in the direction from the vertex $\boldsymbol{v}_{o}$ to vertex $\boldsymbol{v}_{i}$. The associated parametrisation of the edge is given by $\xi_{o i}=\lambda_{i}-\lambda_{o} \in[-1,1]$, as shown in Figure 3(a). The local parametrisation of the edge in the reference element is chosen to coincide with the global parametrisation of the edge.

This process circumvents the sign conflict problem. Consider once again the example illustrated in Figure 1. The common edge $\boldsymbol{e}=[o i]$ would be oriented 


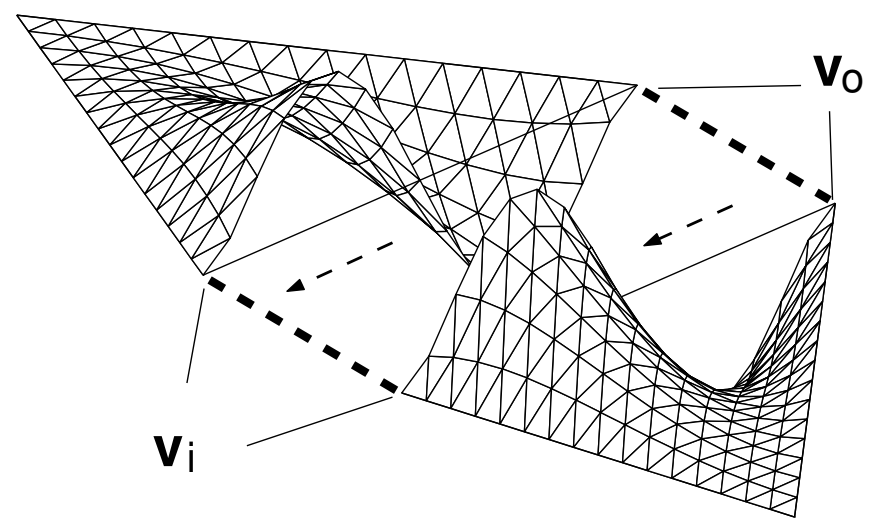

Figure 4: The continuity of the global cubic functions across $e$ is a consequence of a consistent local parametrisation of each edge from the smallest vertex $\boldsymbol{v}_{o}$ to the largest vertex $\boldsymbol{v}_{i}$ on both elements.

in the same direction on both triangles. The local parametrisation $\xi_{o i}$ used to define the local basis functions would then agree on neighbouring elements, and the cubic edge function would automatically be continuous across $\boldsymbol{e}$, as shown in Figure 4. In particular, the constraints (2) would then take the form

$$
\left.\begin{array}{l}
c_{0}=c_{0}^{\prime} \\
c_{1}=c_{1}^{\prime}
\end{array}\right\}
$$

and the issue of sign conflicts no longer arises.

The same notion may be used in three dimensions to circumvent the problem of rotational invariance in enforcing $H^{1}(\Omega)$-conformity across faces. A face $\boldsymbol{f}=[o i j]$ defined by global vertices $\boldsymbol{v}_{o}, \boldsymbol{v}_{i}$ and $\boldsymbol{v}_{j}$ is parameterised by choosing coordinates $\xi_{o i}=\lambda_{i}-\lambda_{o}$ and $\xi_{o j}=\lambda_{j}-\lambda_{o}$, as shown in Figure 3(b). The local parametrisation of the face on the reference element is chosen to agree with the global parametrisation.

Consider once again the situation shown in Figure 2. The face basis functions for the fourth order space are chosen to be of the same form on both elements:

$$
\beta_{\boldsymbol{f}} ; \quad \beta_{\boldsymbol{f}} \xi_{23}=\beta_{\boldsymbol{f}}\left(\lambda_{3}-\lambda_{2}\right) ; \quad \beta_{\boldsymbol{f}} \xi_{24}=\beta_{\boldsymbol{f}}\left(\lambda_{4}-\lambda_{2}\right) .
$$

With this choice of basis, the arguments leading to conditions (5) would yield the trivial set of constraints

$$
c_{0}=c_{0}^{\prime} ; \quad c_{1}=c_{1}^{\prime} ; \quad c_{2}=c_{2}^{\prime},
$$

and conformity is obtained automatically. In short, once a unique global preferred parametrisation of the faces has been defined, the same parametrisation 
may be adopted locally on each element and issues of rotational invariance become irrelevant.

\subsection{Intrinsic Orientation of Edges and Faces}

The foregoing examples illustrate that the orientation of the element faces and edges is the key to enforcing appropriate continuity properties of the discrete spaces. It is important that the method chosen to orient faces and edges can be applied locally on each element in isolation, without the need to refer to exterior entities such as the elements containing them.

Denote the set of vertices, edges and faces of a single element $\boldsymbol{t}$ by $\mathcal{V}(\boldsymbol{t})$, $\mathcal{E}(\boldsymbol{t})$ and $\mathcal{F}(\boldsymbol{t})$, respectively, and the corresponding global sets by $\mathcal{V}, \mathcal{E}$ and $\mathcal{F}$.

Each edge $\boldsymbol{e} \in \mathcal{E}$ is described by a pair $[o i]$ of numbers for the global vertices located at the endpoints of the edge. The ordering of the numbers is determined by requiring $o<i$. The edge may then be assigned a unique parametrisation given by

$$
\xi_{e}: \mathbb{R}^{3} \rightarrow \mathbb{R}: \quad \xi_{e}=\xi_{o i}=\lambda_{i}-\lambda_{o} .
$$

The parametrisation is intrinsic to the edge, depending solely on the global numbering of the endpoints of the edge.

Likewise, a face $f \in \mathcal{F}$ is described by a triple $[o i j]$ formed from the global numbering of the vertices of the face. By applying a rotation of the local numbering of the vertices, it is possible to ensure that the vertex $\boldsymbol{v}_{o}$ with the smallest global numbering, has local number 1 without affecting the properties of the face itself. The ordering of the remaining vertices, $\boldsymbol{v}_{i}$ and $\boldsymbol{v}_{j}$, is then automatically determined so that the cyclic ordering of the vertices is preserved. This process results in a well-defined ordering of the vertices. The face is then assigned a unique parametrisation given by $\xi_{o i}$ and $\xi_{o j}$. Once again, the parametrisation is intrinsic to the face and is determined entirely in terms of the global numbering of the vertices of the face. Equally well, a tetrahedron $\boldsymbol{t}=[o i j k] \in \mathcal{T}$ is parametrised by $\xi_{o i}, \xi_{o j}$ and $\xi_{o k}$, as shown in Figure 3. The ordering of the vertices in the tetrahedron is discussed in detail in the next section.

A unique directed tangent on an edge $\boldsymbol{e}=[o i]$ is given by

$$
\boldsymbol{\tau}^{e}=\boldsymbol{\tau}^{[o i]}=\boldsymbol{v}_{i}-\boldsymbol{v}_{o}
$$

while a unique pair of tangent vectors to face $\boldsymbol{f}=[o i j]$ is defined by

$$
\tau_{1}^{f}=\tau^{[o i]} \text { and } \tau_{2}^{f}=\tau^{[o j]} .
$$

For future reference, we introduce bubble functions on each edge $\boldsymbol{e}$, face $\boldsymbol{f}$ and tetrahedron $\boldsymbol{t}$ by

$$
\beta_{\boldsymbol{e}}=\beta_{o i}=\lambda_{o} \lambda_{i} ; \quad \beta_{\boldsymbol{f}}=\beta_{o i j}=\lambda_{o} \lambda_{i} \lambda_{j} ; \quad \text { and } \beta_{\boldsymbol{t}}=\beta_{o i j k}=\lambda_{o} \lambda_{i} \lambda_{j} \lambda_{k} .
$$

Note that these functions vanish on the boundary of the entity with which they are associated and are therefore continuous functions. 


\subsection{Intrinsic Orientation of Elements}

The intrinsic orientation of the edges and faces described above depends on the global numbering of the vertices. This means that the construction of the basis functions on the reference element also depends on the numbering of the global vertices defining the element. At first sight, this fact appears to carry undesirable consequences. Fortunately, this proves not to be the case thanks to the following key observation: An appropriate reordering of the local numbering to the vertices allows any global tetrahedron to be reduced to one of two possible reference tetrahedra. This process will be illustrated below. The significance of the observation is that the standard finite element technology, whereby computations are performed on a corresponding reference element, is not sacrificed provided two reference tetrahedra are utilised.

The reduction to one of two reference tetrahedra is achieved by the following procedure:

1. Local vertex 1 is aligned with the smallest global vertex $\boldsymbol{v}_{o}$ by rotating the local numbering on either of the two faces containing global vertex $\boldsymbol{v}_{o}$ and local vertex 1. (Of course, if the two are already aligned, then no action is needed.)

2. Local vertex 4 is aligned with the largest global vertex $\boldsymbol{v}_{k}$ by rotating the local numbering on the face opposite local vertex 1.

3. The tetrahedron is then classified as Type I or II, according to the relative ordering of the global numbering of the remaining vertices $\boldsymbol{v}_{i}$ and $\boldsymbol{v}_{j}$. If $i<j$, then the tetrahedron is of Type I, and otherwise of Type II (see Figure 5).

As a simple example, consider a tetrahedron with global numbering [15 96824 ] as shown in Figure 6(a). The general procedure applied to this particular tetrahedron gives:

1. The triple [15968] representing a face containing the smallest global and local vertices, is cyclically permuted until the smallest global vertex has local number 1 . This yields [81596] and is equivalent to the rotation of a local face to align the smallest global and local vertices as shown in Figure 6(b). This gives the new local to global numbering [8 159624 ] for the tetrahedron.

2. The triple [159624] representing the face opposite the smallest local vertex, is cyclically permuted to align global vertex 96 with local vertex 4 . This is equivalent to rotating the local face opposite the smallest local vertex as shown in Figure 6(c). This results in a new local to global mapping [8 241596$]$.

3. This tetrahedron is then classified as a Type II reference element as shown in Figure 5 (because $24>15$ ). 


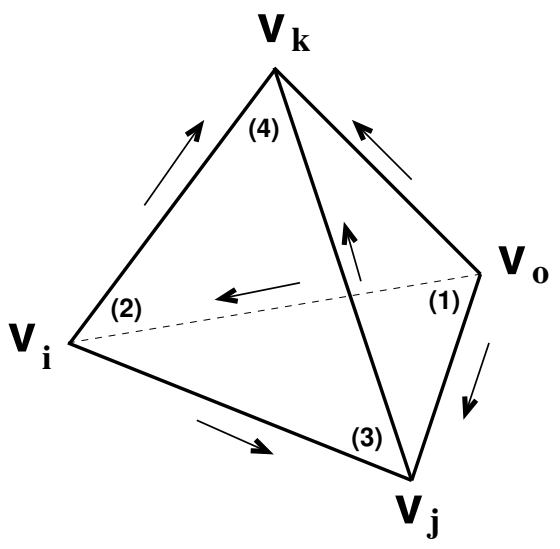

(a) Type I: $i<j$

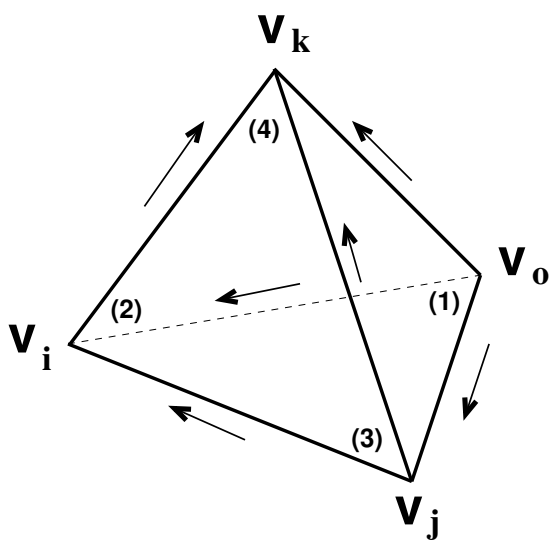

(b) Type II: $i>j$

Figure 5: The two possible reference tetrahedra. The arrows indicate the global orientation of the edges. The tetrahedra differ only in the orientation of the edge connecting vertices $\boldsymbol{v}_{i}$ and $\boldsymbol{v}_{j}$, which depends on the ordering of the global numbers $i$ and $j$. If $i<j$, then the tetrahedron is of Type I, and of Type II otherwise.

If the original numbering of the tetrahedron is given by [2496815], then the procedure would result in a numbering given by [8 152496] corresponding to a Type I reference element.

In practice, the reduction to a Type I or II reference configuration is performed as a pre-processing step at a negligible cost. The fact that every element may be reduced to a Type I or II reference element is of vital significance: $E v$ ery edge and every face of the appropriate reference configuration is identical with the intrinsic orientations of the edges and faces viewed in isolation. This means that the parametrisation on the reference element will automatically be compatible with the global parametrisation of the edges and faces induced by the intrinsic orientation. Consequently, once the tetrahedra have been classified as Type I or II, there is no need to check orientations during the finite element analysis.

\section{Hierarchic Bases}

Hierarchic basis functions are essential for the efficient, practical implementation of higher order finite element methods, such as $p$ and $h p$-version procedures, where increased accuracy relies on increasing the order of the polynomial space. Here, we derive hierarchic bases for arbitrary, non-uniform order discretisation of the spaces $H^{1}, \boldsymbol{H}$ (curl), $\boldsymbol{H}(\mathbf{d i v})$ and $L_{2}$ on an unstructured partitioning $\mathcal{T}$ of the domain into tetrahedral elements. It is assumed throughout that the partitioning is regular in the sense that the non-empty intersection of distinct elements is either a single common vertex, edge or face of both elements. Non-degenerate curvilinear elements are permitted. In particular, each element 




(a) Original numbering of physical tetrahedron [1596824].

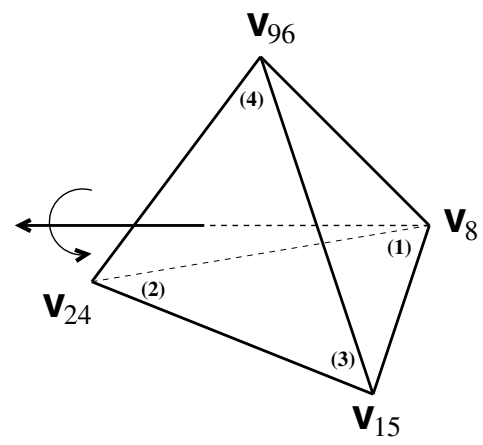

(c) Align vertices with greatest local and global numbers by rotating the face containing both vertices giving the new numbering [8 241596$]$.

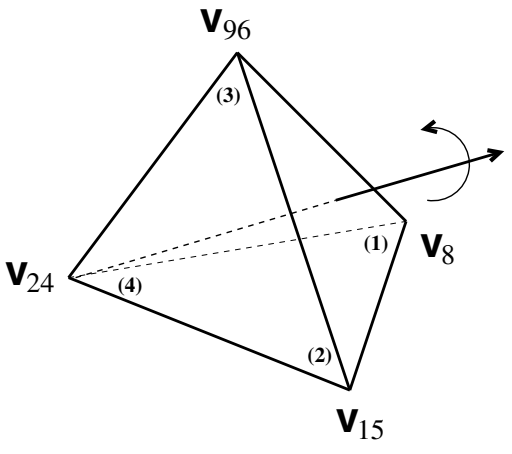

(b) Align vertices with smallest local and global numbers by rotating a face containing both vertices, giving the new numbering [8 159624$]$.

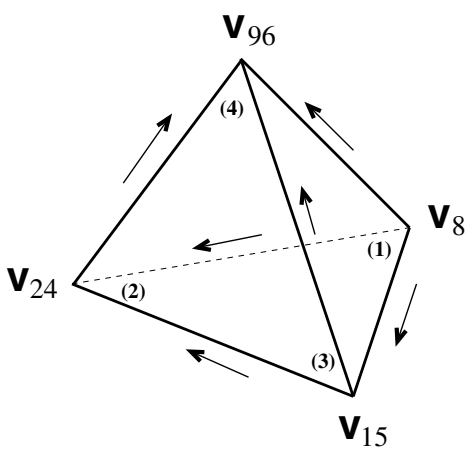

(d) Final alignment of tetrahedron and orientation of edges. The relative ordering of the global numbers for local nodes 2 and 3 corresponds to a Type II element.

Figure 6: Reduction of tetrahedron with original numbering [1596 824 ] to Type II reference tetrahedron. The local numbers are shown in parentheses. 
$\boldsymbol{t} \in \mathcal{T}$ is assumed to be the image of a reference tetrahedron $\hat{\boldsymbol{t}}$ under a smooth bijective mapping $\boldsymbol{F}_{\boldsymbol{t}}$, with the Jacobian of the mapping denoted by $\boldsymbol{J}_{\boldsymbol{t}}$.

A set $\left\{\psi_{\ell}: \ell=0,1, \ldots\right\}$ constitutes a hierarchic basis on a reference interval $I=[-1,1]$ if, for each $\ell=0,1, \ldots$, the function $\psi_{\ell}$ is a polynomial of degree $\ell$. This set will be used to construct general bases on tetrahedra and is referred to as the primary basis. One obvious choice for $\psi_{\ell}$ would be the monomial of degree $\ell$. However, in practice, $\psi_{\ell}$ is often taken to be the Legendre polynomial $L_{\ell}$ of degree $\ell$, defined inductively by the relation

$$
\left.\begin{array}{l}
L_{0}(s)=1 ; \quad L_{1}(s)=s ; \\
L_{\ell+1}(s)=\frac{2 \ell+1}{\ell+1} s L_{\ell}(s)-\frac{\ell}{\ell+1} L_{l-1}(s), \quad \ell=1,2, \ldots
\end{array}\right\}
$$

As a matter of fact, any family of polynomials could be chosen, such as the Gegenbauer polynomials [15, Section 8.93] defined, for fixed $\alpha$, by

$$
\left.\begin{array}{l}
\psi_{0}(s)=1 ; \quad \psi_{1}(s)=2 \alpha s \\
\psi_{\ell+1}(s)=\frac{2(\alpha+\ell)}{\ell+1} s \psi_{\ell}(s)-\frac{2 \alpha+\ell-1}{\ell+1} \psi_{\ell-1}(s), \quad \ell=1,2, \ldots
\end{array}\right\}
$$

The choice $\alpha=\frac{1}{2}$ gives the Legendre polynomials.

The primary basis for an interval $I$ may be used to construct polynomial bases on edges, faces and interiors by taking the arguments of the primary basis functions to be of the form

$$
\xi_{o i}=\lambda_{i}-\lambda_{o} \in[-1,1]
$$

as follows:

Lemma 1 Let $\left\{\psi_{\ell}: \ell=0,1, \ldots\right\}$ be a hierarchic basis on a reference interval I. Then, bases for the spaces $\mathbb{P}_{p}$ of polynomials of total degree at most $p \in \mathbb{N}$ on edges, faces and interiors are given by

1. for $\boldsymbol{e}=[o i] \in \mathcal{E}$,

$$
\mathbb{P}_{p}(\boldsymbol{e})=\operatorname{span}\left\{\psi_{\ell}\left(\xi_{o i}\right): 0 \leq \ell \leq p\right\}
$$

2. for $\boldsymbol{f}=[o i j] \in \mathcal{F}$,

$$
\mathbb{P}_{p}(\boldsymbol{f})=\operatorname{span}\left\{\psi_{\ell}\left(\xi_{o i}\right) \psi_{m}\left(\xi_{o j}\right): 0 \leq \ell, m, \ell+m \leq p\right\}
$$

3. for $\boldsymbol{t}=[$ oij $k] \in \mathcal{T}$,

$$
\mathbb{P}_{p}(\boldsymbol{t})=\operatorname{span}\left\{\psi_{\ell}\left(\xi_{o i}\right) \psi_{m}\left(\xi_{o j}\right) \psi_{n}\left(\xi_{o k}\right): 0 \leq \ell, m, n, \ell+m+n \leq p\right\} .
$$

The proof of this result is left as an elementary exercise. For future reference it is worth noting that the functions in each of these sets are linearly independent. 
Space: $\mathbb{P}_{p}(\hat{\boldsymbol{t}}), p \in \mathbb{N}$

Interior Functions: $\hat{\boldsymbol{t}}=[o i j k]$

$\hat{\varphi}_{\ell m n}^{\hat{\boldsymbol{t}}}=\psi_{\ell}\left(\hat{\xi}_{o i}\right) \psi_{m}\left(\hat{\xi}_{o j}\right) \psi_{n}\left(\hat{\xi}_{o k}\right), \quad 0 \leq \ell, m, n, \ell+m+n \leq p$

Table 1: Hierarchic basis functions for $L_{2}$-conforming finite element space of order $p$.

\section{1 $L_{2}$ and $H^{1}$-Conforming Basis Functions}

A conforming discretisation of the space $H^{1}(\Omega)$ is characterised by continuity (of traces) across element interfaces. By way of contrast, the space $L_{2}$ imposes no such continuity requirements on interfaces which simplifies the construction of conforming finite element spaces dramatically.

\subsubsection{Reference Element Basis Functions}

The polynomial spaces of order $p$ on the reference element $\hat{\boldsymbol{t}}$ for both the $H^{1}$ and $L_{2}$ spaces is given by

$$
\hat{X}_{p}^{H^{1}}=\hat{X}_{p}^{L_{2}}=\mathbb{P}_{p}(\hat{\boldsymbol{t}}) .
$$

However, the differing continuity requirements on the interfaces mean that different bases are used to realise these spaces. For instance, the set of hierarchic basis functions for $\hat{X}_{p}^{L_{2}}$ given in Table 1 consists entirely of interior functions. The term interior reflects the nature of the degrees of freedom associated with the functions. The absence of conformity conditions for the space $L_{2}$ means that the coefficients of all basis functions can be chosen completely independently on all elements. In this sense, the degrees of freedom are internal to the element.

Conversely, the set of hierarchic basis functions for $\hat{X}_{p}^{H^{1}}$ based on the primary basis $\left\{\psi_{\ell}: \ell \in \mathbb{N}\right\}$ given in Table 2 is split into sets of functions identified with element vertices, edges, faces and interiors. This reflects the differing conformity conditions for the space $H^{1}$. The requirement for continuity between elements means that the basis functions must match at vertices, on edges and on faces. Vertex functions are the only non-zero functions at element vertices and therefore must have the same coefficient on all elements containing the vertex. Similarly, edge functions are (apart from the vertex functions) the only non-zero functions on element edges and must have identical coefficients on all elements containing the edge. Equally well, the coefficients of the face functions need only match on the pair of elements sharing the common face. Finally, interior functions vanish on all shared boundaries and their coefficients may be chosen independently on each element.

It will be observed that the nature of a function depends on the particular space under consideration. The same function could be an interior function for 


$$
\text { Space: } \mathbb{P}_{p}(\hat{\boldsymbol{t}}), p \in \mathbb{N}
$$

Vertex Functions: $\boldsymbol{v} \in \mathcal{V}(\hat{\boldsymbol{t}})$

$$
\hat{\varphi}^{v}=\hat{\lambda}_{v}
$$

$\underline{\text { Edge Functions: }} \boldsymbol{e}=[o i] \in \mathcal{E}(\hat{\boldsymbol{t}})$

$$
\hat{\varphi}_{\ell}^{e}=\beta_{o i} \psi_{\ell}\left(\hat{\xi}_{o i}\right), \quad 0 \leq \ell \leq p-2
$$

Face Functions: $\boldsymbol{f}=[o i j] \in \mathcal{F}(\hat{\boldsymbol{t}})$

$$
\hat{\varphi}_{\ell m}^{f}=\beta_{o i j} \psi_{\ell}\left(\hat{\xi}_{o i}\right) \psi_{m}\left(\hat{\xi}_{o j}\right), \quad 0 \leq \ell, m, \ell+m \leq p-3
$$

Interior Functions: $\hat{\boldsymbol{t}}=[o i j k]$

$$
\begin{array}{r}
\hat{\varphi}_{\ell m n}^{\hat{t}}=\beta_{o i j k} \psi_{\ell}\left(\hat{\xi}_{o i}\right) \psi_{m}\left(\hat{\xi}_{o j}\right) \psi_{n}\left(\hat{\xi}_{o k}\right), \\
0 \leq \ell, m, n, \ell+m+n \leq p-4
\end{array}
$$

Table 2: Hierarchic basis functions for $H^{1}$-conforming finite element space of order $p$.

the $L_{2}$ case but need not necessarily be an interior function for the $H^{1}$ space. For instance, an interior function for the space $L_{2}$ need not vanish on element boundaries.

Lemma 2 The set of functions defined in Tables 1 and 2 forms a hierarchic basis for the spaces $\hat{X}_{p}^{H^{1}}$ and $\hat{X}_{p}^{L_{2}}, p \in \mathbb{N}$, respectively.

Proof. Let $p \in \mathbb{N}$. The result in case of the space $L_{2}$ is an immediate consequence of Lemma 1. The $H^{1}$ case requires some attention. The vertex function $\hat{\varphi}^{\boldsymbol{v}}$ belongs to $\mathbb{P}_{1}$ and, a fortiori, $\mathbb{P}_{p}$. Consider an edge function $\hat{\varphi}_{\ell}^{e}, \ell \in\{0, \ldots, p-2\}$. By definition, $\psi_{\ell}$ is a degree $\ell$ polynomial, and so, since $\xi_{i j}=\lambda_{j}-\lambda_{i} \in \mathbb{P}_{1}$, it follows that the function $\psi_{\ell}\left(\xi_{i j}\right)$ belongs to $\mathbb{P}_{\ell}$. Consequently, the edge function $\hat{\varphi}_{\ell}^{e}$ belongs to $\mathbb{P}_{\ell+2}$ and hence also belongs to $\mathbb{P}_{p}$ since $\ell \leq p-2$. Similar arguments show that the face and interior functions also belong to the space $\mathbb{P}_{p}$.

The functions are linearly independent. For instance, suppose

$$
\sum_{\boldsymbol{v} \in \mathcal{V}} w_{\boldsymbol{v}}+\sum_{\boldsymbol{e} \in \mathcal{E}} w_{\boldsymbol{e}}+\sum_{\boldsymbol{f} \in \mathcal{F}} w_{\boldsymbol{f}}+w_{\boldsymbol{t}} \equiv 0
$$

where $w_{\boldsymbol{v}} \in \operatorname{span}\left\{\hat{\varphi}^{\boldsymbol{v}}\right\}$ etc. The presence of the edge, face and interior bubble functions in the definitions means that $\sum_{\boldsymbol{v} \in \mathcal{V}} w_{\boldsymbol{v}}$ vanishes at the element vertices. Consequently, since the barycentric coordinate functions are linearly independent, each term $w_{\boldsymbol{v}}$ vanishes and it follows that the first term in equation (12) is identically zero. The only remaining term in (12) that is non-zero 
on the element edge $\boldsymbol{e}$ is $w_{\boldsymbol{e}} \in \beta_{\boldsymbol{e}} \operatorname{span}\left\{\psi_{\ell}\right\}$. It follows that $w_{\boldsymbol{e}}$ must vanish identically since $\left\{\psi_{\ell}\right\}$ forms a basis on an interval. Consequently, the second term in (12) vanishes. Similarly, $w_{\boldsymbol{f}}$ vanishes on the face $\boldsymbol{f}$, and analogous arguments show $w_{\boldsymbol{f}}$ must therefore be identically zero. Finally, it follows that $w_{\boldsymbol{t}}$ vanishes on the element interior, and arguing as above, is therefore identically zero.

The proof is concluded by observing that the number of functions defined in Table 2 coincides with the dimension, $(p+1)(p+2)(p+3) / 6$, of the space $\mathbb{P}_{p}$.

Sets of basis functions similar to those given in Table 2 are well-known in the literature. For instance, the basis presented in [32] differ only in the choice of local parametrisation used to define the basis functions associated with the faces and interior.

\subsubsection{Global Basis Functions}

A global basis function $\varphi$ defined on a physical element $t \in \mathcal{T}$ is constructed from the basis function $\hat{\varphi}$ defined on the reference element using the standard pull-back transformation:

$$
\left.\varphi\right|_{t}=\hat{\varphi} \circ \boldsymbol{F}_{\boldsymbol{t}}^{-1} .
$$

The global discrete $L_{2}(\Omega)$ space of order $p$ is given by

$$
X_{p, \mathcal{T}}^{L_{2}}=\bigoplus_{\boldsymbol{t} \in \mathcal{T}} \operatorname{span}\left\{\varphi_{\ell, m, n}^{\boldsymbol{t}}: 0 \leq \ell, m, n \leq p\right\}
$$

while the corresponding $H^{1}(\Omega)$ space is defined by

$$
\begin{aligned}
X_{p, \mathcal{T}}^{H^{1}}= & \bigoplus_{\boldsymbol{v} \in \mathcal{V}} \operatorname{span}\left\{\varphi^{\boldsymbol{v}}\right\} \\
& \bigoplus_{\boldsymbol{e} \in \mathcal{E}} \operatorname{span}\left\{\varphi_{\ell}^{\boldsymbol{e}}: 0 \leq \ell \leq p-2\right\} \\
& \bigoplus_{\boldsymbol{f} \in \mathcal{F}} \operatorname{span}\left\{\varphi_{\ell, m}^{\boldsymbol{f}}: 0 \leq \ell, m \leq p-3\right\} \\
& \bigoplus_{\boldsymbol{t} \in \mathcal{T}} \operatorname{span}\left\{\varphi_{\ell, m, n}^{\boldsymbol{t}}: 0 \leq \ell, m, n \leq p-4\right\} .
\end{aligned}
$$

The next result shows that these are conforming subspaces:

Theorem 3 The spaces $X_{p, \mathcal{T}}^{L_{2}}$ and $X_{p, \mathcal{T}}^{H^{1}}$ are $L_{2}$-conforming and $H^{1}$-conforming subspaces of order $p$, respectively.

Proof. The case of the space $L_{2}$ is trivial since no conformity conditions are required, so restrict attention to the space $H^{1}$. Lemma 2 shows that $\hat{X}_{p, \mathcal{T}}^{H^{1}}$ corresponds to an order $p$ space and it suffices to demonstrate $H^{1}$-conformity.

Let $\boldsymbol{t}, \boldsymbol{t}^{\prime} \in \mathcal{T}$ be distinct, intersecting tetrahedra. There are three cases to be considered: 
Case (i): If $\boldsymbol{t} \cap \boldsymbol{t}^{\prime}$ is a single common vertex $\boldsymbol{v}$, then the only basis function that is non-zero on both elements is the vertex function $\varphi^{v}=\lambda_{\boldsymbol{v}}$, which, being a barycentric coordinate function, is globally continuous.

Case (ii): If $\boldsymbol{t} \cap \boldsymbol{t}^{\prime}$ is a single common edge $\boldsymbol{e}=[o i]$, then, apart from the vertex functions, the edge functions $\varphi_{\ell}^{e}$ are non-zero on both elements. The restriction of the edge basis function to each element is given by $\beta_{o i} \psi\left(\xi_{o i}\right)$. Since both $\beta_{o i}=\lambda_{o} \lambda_{i}$ and $\xi_{o i}=\lambda_{i}-\lambda_{o}$ are globally continuous and depend only on the vertex numbers of the edge, it follows that the edge function is continuous across interfaces between all elements containing the edge.

Case (iii): If $\boldsymbol{t} \cap \boldsymbol{t}^{\prime}$ is a single common face $\boldsymbol{f}=[o i j]$, then apart from the vertex and edge functions (already shown to be continuous), the face functions $\varphi_{\ell m}^{f}$ are non-zero on both elements. The restriction of the face function to both elements is given by $\beta_{o i j} \psi_{\ell}\left(\xi_{o i}\right) \psi_{m}\left(\xi_{o j}\right)$, and, arguing as before, these are smooth functions of the globally continuous barycentric coordinate functions. Thus, the face functions are continuous across the shared interface.

The interior functions are supported on a single element, and are therefore trivially seen to be globally continuous.

\section{2 $H$ (curl)-Conforming Basis Functions}

A conforming discretisation of the space $\boldsymbol{H}$ (curl) is characterised by continuity of tangential components across element interfaces. More precisely, Nedelec [29, Lemma 3] shows that if domains $K$ and $K^{\prime}$ share a common face $\boldsymbol{f}$ with normal $\boldsymbol{n}$, then a smooth vector field $\boldsymbol{u}$ on each domain belongs to $\boldsymbol{H}$ (curl; $K \cup K^{\prime}$ ) provided that $\boldsymbol{n} \wedge \boldsymbol{u}$ is the same on each side of the face $\boldsymbol{f}$.

\subsubsection{Reference Element Basis Functions}

The vector-valued polynomial space of order $p$ on the reference element $\hat{\boldsymbol{t}}$ is given by

$$
\hat{\boldsymbol{X}}_{p}^{\text {curl }}=\left(\mathbb{P}_{p}(\hat{\boldsymbol{t}})\right)^{3} .
$$

A set of hierarchic basis functions for $\hat{\boldsymbol{X}}_{p}^{\text {curl }}$ is given in Table 3 .

The lowest order edge function $\hat{\varphi}_{0}^{e}$ defined in Table 3 coincides with the so-called Whitney element $[7,36]$. The two lowest order edge functions have the property that on an edge $\boldsymbol{e}^{\prime}$,

$$
\left.\hat{\boldsymbol{\tau}}_{\boldsymbol{e}} \cdot \hat{\boldsymbol{\varphi}}_{0}^{e}\right|_{\boldsymbol{e}^{\prime}}=\left\{\begin{array}{ll}
1 & \text { if } \boldsymbol{e}=\boldsymbol{e}^{\prime} \\
0 & \text { otherwise }
\end{array} \text { and }\left.\hat{\boldsymbol{\tau}}_{\boldsymbol{e}} \cdot \hat{\boldsymbol{\varphi}}_{1}^{e}\right|_{\boldsymbol{e}^{\prime}}=\left\{\begin{aligned}
\xi_{\boldsymbol{e}} & \text { if } \boldsymbol{e}=\boldsymbol{e}^{\prime} \\
0 & \text { otherwise. }
\end{aligned}\right.\right.
$$

These relations may be generalised by using the recurrence relation (10) defining the Legendre polynomials to reveal that the edge functions defined in Table 3 satisfy, for $\ell=0,1,2, \ldots$,

$$
\left.\hat{\tau}_{\boldsymbol{e}} \cdot \hat{\varphi}_{\ell}^{e}\right|_{e^{\prime}}= \begin{cases}L_{\ell}\left(\xi_{\boldsymbol{e}}\right) & \text { if } \boldsymbol{e}=\boldsymbol{e}^{\prime} \\ 0 & \text { otherwise }\end{cases}
$$




\section{Space: $\left(\mathbb{P}_{p}(\hat{\boldsymbol{t}})\right)^{3}, p \in \mathbb{N}$}

$\underline{\text { Edge Functions: }} \boldsymbol{e}=[o i] \in \mathcal{E}(\hat{\boldsymbol{t}})$

$$
\begin{aligned}
\hat{\varphi}_{0}^{e} & =\hat{\lambda}_{i} \hat{\nabla} \hat{\lambda}_{o}-\hat{\lambda}_{o} \hat{\nabla} \hat{\lambda}_{i} \\
\hat{\varphi}_{1}^{e} & =\hat{\lambda}_{i} \hat{\nabla} \hat{\lambda}_{o}+\hat{\lambda}_{o} \hat{\nabla} \hat{\lambda}_{i} \\
\hat{\varphi}_{\ell+1}^{e} & =\frac{2 \ell+1}{\ell+1} L_{\ell}\left(\hat{\xi}_{o i}\right) \hat{\varphi}_{1}^{e}-\frac{\ell}{\ell+1} L_{\ell-1}\left(\hat{\xi}_{o i}\right) \hat{\varphi}_{0}^{e}, \quad 2 \leq \ell \leq p-1
\end{aligned}
$$

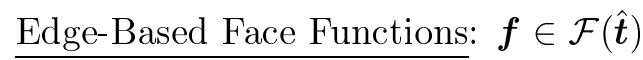

For each edge $\boldsymbol{e} \subset \partial \boldsymbol{f}$ :

$$
\hat{\varphi}_{\boldsymbol{e}, \ell}^{\boldsymbol{f}}=\beta_{\boldsymbol{e}} \psi\left(\hat{\xi}_{\boldsymbol{e}}\right) \hat{\nabla} \hat{\lambda}_{\boldsymbol{f} \backslash \boldsymbol{e}}, \quad 0 \leq \ell \leq p-2,
$$

where $\boldsymbol{f} \backslash \boldsymbol{e}$ denotes the vertex opposite edge $\boldsymbol{e}$ in face $\boldsymbol{f}$.

Face Bubble Functions: $\boldsymbol{f}=[o i j] \in \mathcal{F}(\hat{\boldsymbol{t}})$

$$
\left.\begin{array}{l}
\hat{\boldsymbol{\varphi}}_{i, \ell m}^{\boldsymbol{f}}=\beta_{o i j} \psi_{\ell}\left(\hat{\xi}_{o i}\right) \psi_{m}\left(\hat{\xi}_{o j}\right) \hat{\boldsymbol{\tau}}^{[o i]} \\
\hat{\boldsymbol{\varphi}}_{j, \ell m}^{\boldsymbol{f}}=\beta_{o i j} \psi_{\ell}\left(\hat{\xi}_{o i}\right) \psi_{m}\left(\hat{\xi}_{o j}\right) \hat{\boldsymbol{\tau}}^{[o j]}
\end{array}\right\} \quad 0 \leq \ell, m, \ell+m \leq p-3
$$

Face-Based Interior Functions: $\boldsymbol{f}=[o i j] \in \mathcal{F}(\hat{\boldsymbol{t}})$

$$
\hat{\boldsymbol{\varphi}}_{\boldsymbol{f}, \ell m}^{\hat{\boldsymbol{t}}_{\ell}}=\beta_{\boldsymbol{f}} \psi_{\ell}\left(\hat{\xi}_{o i}\right) \psi_{m}\left(\hat{\xi}_{o j}\right) \hat{\nabla} \hat{\lambda}_{\boldsymbol{t} \backslash \boldsymbol{f}}, \quad 0 \leq \ell, m, \ell+m \leq p-3
$$

where $\boldsymbol{t} \backslash \boldsymbol{f}$ denotes the vertex of $\hat{\boldsymbol{t}}$ opposite face $\boldsymbol{f}$.

Interior Bubble Functions: $\hat{\boldsymbol{t}}=[o i j k]$

$$
\begin{array}{r}
\hat{\boldsymbol{\varphi}}_{d, \ell m n}^{\hat{\boldsymbol{t}}}=\beta_{\boldsymbol{t}} \psi_{\ell}\left(\hat{\xi}_{o i}\right) \psi_{m}\left(\hat{\xi}_{o j}\right) \psi_{n}\left(\hat{\xi}_{o k}\right) \hat{\boldsymbol{e}}_{d}, \quad d \in\{1,2,3\} \\
0 \leq \ell, m, n, \ell+m+n \leq p-4
\end{array}
$$

Table 3: Hierarchic basis functions for $\boldsymbol{H}$ (curl)-conforming finite element space of order $p$. 
Altogether, there are $(p+1)$ edge basis functions associated with each edge of the reference element, giving $6(p+1)$ in total.

The face functions are more complicated and fall into two categories: Edgebased face functions $\hat{\varphi}_{\boldsymbol{e}, \ell}^{\boldsymbol{f}}$ are associated with the edges $\boldsymbol{e}$ of face $\boldsymbol{f}$ while face bubble functions are associated with the face $\boldsymbol{f}$ itself. The key distinction is that face bubble functions on $\boldsymbol{f}$ vanish on all other faces while edge-based face functions are also non-zero on the face $\boldsymbol{f}^{\prime}$ sharing an edge $\boldsymbol{e}$ with $\boldsymbol{f}$. However, since the vector $\hat{\nabla} \hat{\lambda}_{\boldsymbol{f} \backslash \boldsymbol{e}}$ is normal to the face $\boldsymbol{f}^{\prime}$, the tangential components of $\hat{\varphi}_{e, \ell}^{f}$ are non-zero only on the face $\boldsymbol{f}$ itself. The conformity conditions characterising the space $\boldsymbol{H}$ (curl) depend only on tangential components and therefore, the degrees of freedom for the edge-based face functions are associated with a single face. This justifies classifying these as face functions.

The tangential components of the edge-based functions on face $f$ satisfy,

$$
\left.\boldsymbol{n}^{\boldsymbol{f}} \wedge \hat{\varphi}_{\boldsymbol{e}, \ell}^{\boldsymbol{f}}\right|_{\boldsymbol{f}}=c \beta_{e} \psi_{\ell}\left(\xi_{e}\right) \boldsymbol{\tau}_{\boldsymbol{e}},
$$

which follows from the observation that

$$
n^{f} \wedge \hat{\nabla} \hat{\lambda}_{f \backslash e}=c \tau_{e}
$$

where $c$ is the (non-zero) determinant of the matrix whose columns are formed from the components of the constant vectors $\tau_{\boldsymbol{e}}, \boldsymbol{n}^{\boldsymbol{f}}$ and $\hat{\nabla} \hat{\lambda}_{\boldsymbol{f} \backslash \boldsymbol{e}}$. Altogether, there are $3(p-1)$ edge-based face functions and $(p-1)(p-2)$ face bubble functions for each face of the reference tetrahedron, giving $4(p-1)(p+1)$ face functions in total.

The interior functions also fall into two categories: Interior bubble functions functions vanish on all faces of the tetrahedron while face-based interior functions are non-zero on certain faces. However, the tangential components of the face-based functions do vanish on all faces of the tetrahedron. Nevertheless, the classification as interior functions is justified since $\boldsymbol{H}$ (curl)-conformity imposes conditions on tangential components only, meaning that the coefficients of the face-based functions can be chosen independently on each element.

There are $(p-1)(p-2)(p-3) / 2$ interior bubble functions and $(p-1)(p-$ $2) / 2$ face-based interior functions for each face of the reference element, giving $(p-1)(p-2)(p+1) / 2$ in total.

Lemma 4 The set of functions defined in Table 3 forms a hierarchic basis for the spaces $\hat{\boldsymbol{X}}_{p}^{\text {curl }}, p \in \mathbb{N}$.

Proof. Observe that each of the functions defined in Table 3 belongs to the space $\left(\mathbb{P}_{p}\right)^{3}$. The next step is to show the functions are linearly independent. Suppose that

$$
\sum_{e \in \mathcal{E}} \boldsymbol{w}^{e}+\sum_{f} \sum_{e \subset \partial f} w_{e}^{f}+\sum_{f} w^{f}+\sum_{f} w_{f}^{t}+w^{t} \equiv 0
$$

where $\boldsymbol{w}^{e}, \boldsymbol{w}^{f}, \boldsymbol{w}_{e}^{f}, \boldsymbol{w}_{f}^{t}$ and $\boldsymbol{w}^{t}$ are linear combinations of edge, face and interior functions, respectively. With the aid of Lemma 1, it is not difficult to 
show that the basis functions within these categories are linearly independent, and it therefore suffices to show (16) implies the each of the above functions must vanish individually.

Let $e \in \mathcal{E}$ be any edge, then, taking inner product of (16) with $\tau_{e}$ on the edge gives

$$
\left.\tau_{e} \cdot w^{e}\right|_{e}=0
$$

since the tangential components of the face and interior functions vanish on all edges. Property (14) then implies that $\boldsymbol{w}^{e}=\mathbf{0}$ and it follows that the first term in (16) vanishes.

Let $\boldsymbol{f} \in \mathcal{F}$ be a face containing the edge $\boldsymbol{e}$, then, forming the vector product of (16) with $\boldsymbol{n}^{\boldsymbol{f}}$ on the face $\boldsymbol{f}$ gives

$$
\left.n^{f} \wedge w_{e}^{f}\right|_{f}+\left.n^{f} \wedge w^{f}\right|_{f}=0
$$

since the tangential components of interior functions and the face bubble functions vanish on all faces. Restricting (17) to an edge of face $\boldsymbol{f}$ and recalling the face bubble functions vanish on the edges, and then applying property (15) shows that the edge-based face function $\boldsymbol{w}_{e}^{f}=\mathbf{0}$ and it follows that the second term in (16) must vanish. Equation (17) then implies the third term vanishes too.

Similarly, taking inner products of (16) with $\boldsymbol{n}^{\boldsymbol{f}}$ on the face $\boldsymbol{f}$ gives

$$
\left.\boldsymbol{n}^{\boldsymbol{f}} \cdot \boldsymbol{w}_{\boldsymbol{f}}^{t}\right|_{\boldsymbol{f}}=0
$$

since the interior bubble functions vanish on all faces. Inserting the expressions for the face-based interior functions and noting that the gradient is normal to the face leads to the conclusion $\boldsymbol{w}_{\boldsymbol{f}}^{t}=\mathbf{0}$ and the third term in equation (16) drops out. Consequently, equation (16) reduces to $\boldsymbol{w}^{t}=\mathbf{0}$ and it follows that the basis functions are linearly independent.

The proof is concluded by observing that the number of basis functions defined in Table 3 coincides with the dimension, $(p+1)(p+2)(p+3) / 2$, of the space $\left(\mathbb{P}_{p}\right)^{3}$.

\subsubsection{Global Basis Functions}

A global basis function $\varphi$ of $\boldsymbol{H}$ (curl) defined on a physical element $\boldsymbol{t} \in \mathcal{T}$ is constructed from the basis function $\hat{\varphi}$ defined on the reference element using a covariant transformation:

$$
\left.\varphi\right|_{t}=\left(\boldsymbol{J}_{t}^{-\top} \hat{\varphi}\right) \circ \boldsymbol{F}_{\boldsymbol{t}}^{-1} .
$$

The global discrete $\boldsymbol{H}(\mathbf{c u r l})$ space of order $p$ is defined by

$$
\begin{aligned}
\boldsymbol{X}_{p, \mathcal{T}}^{\text {curl }}= & \bigoplus_{\boldsymbol{e} \in \mathcal{E}} \quad \operatorname{span}\left\{\varphi_{\ell}^{e}: 0 \leq \ell \leq p\right\} \\
& \bigoplus_{\boldsymbol{f} \in \mathcal{F}} \quad \operatorname{span}\left\{\varphi_{\boldsymbol{e}, \ell}^{\boldsymbol{f}}: 0 \leq \ell \leq p-2, \boldsymbol{e} \subset \partial \boldsymbol{f}\right\}
\end{aligned}
$$




$$
\begin{array}{ll}
\bigoplus_{\boldsymbol{f}=[o i j] \in \mathcal{F}} & \operatorname{span}\left\{\boldsymbol{\varphi}_{i, \ell m}^{\boldsymbol{f}}, \boldsymbol{\varphi}_{j, \ell m}^{\boldsymbol{f}}: 0 \leq \ell, m \leq p-3\right\} \\
\bigoplus_{\boldsymbol{t} \in \mathcal{T}} & \operatorname{span}\left\{\boldsymbol{\varphi}_{\boldsymbol{f}, \ell m}^{\boldsymbol{t}}: 0 \leq \ell, m \leq p-3, \boldsymbol{f} \subset \partial \boldsymbol{t}\right\} \\
\bigoplus_{\boldsymbol{t} \in \mathcal{T}} & \operatorname{span}\left\{\boldsymbol{\varphi}_{d, \ell m n}^{\boldsymbol{t}}: d \in\{1,2,3\}, 0 \leq \ell, m, n \leq p-4\right\} .
\end{array}
$$

The main result of this section reads as follows:

Theorem $\mathbf{5}$ The space $\boldsymbol{X}_{p, \mathcal{T}}^{\text {curl }}$ is an $\boldsymbol{H}$ (curl)-conforming subspace of order $p$.

Proof. Let $p \in \mathbb{N}$ be fixed. Lemma 4 shows that the space is of $p$-th order. It only remains to show that if distinct elements $\boldsymbol{t}$ and $\boldsymbol{t}^{\prime}$ share a common face $\boldsymbol{f}$, then for each global basis function, the quantity $n^{f} \wedge \varphi$ is continuous across the face $f$.

Edge functions: Let $\boldsymbol{e} \subset \partial \boldsymbol{f}$ be an edge of the shared face. The higher order edge functions $\hat{\varphi}_{\ell}^{e}$ are combinations of functions of $\xi_{e}$ multiplied by the two lowest order edge functions, which, in turn, are combinations of the gradients of the barycentric coordinates on the edge. The coordinate $\xi_{e}$ is continuous on the shared face, and it therefore suffices to show that $\boldsymbol{n}^{\boldsymbol{f}} \wedge \boldsymbol{J}_{t}^{-\top} \hat{\nabla} \hat{\lambda}$ is continuous across the face, where $\hat{\lambda}$ is a barycentric coordinate on the face. The chain rule confirms that $\boldsymbol{J}_{t}^{-\top} \hat{\nabla} \hat{\lambda}$ is simply the gradient with respect to the physical coordinates, $\nabla \lambda$. The barycentric coordinate functions have continuous tangential components across shared faces, and therefore $\boldsymbol{n}^{f} \wedge \nabla \lambda$ is continuous across the interface.

Face functions: The tangential components of both types of face function are only non-zero on the face $\boldsymbol{f}$ with which they are associated. On the face, the edge-based face functions are combinations of functions of the barycentric coordinates on the face and their gradients. Consequently, arguing as in the case of edge functions, we conclude that the tangential components are continuous across the interface. The face bubble functions are also functions of the barycentric coordinates on the face and the two intrinsic tangent vectors $\tau_{i}^{f}$, $\tau_{j}^{f}$ defined on the face. Consequently, since the tangent vectors coincide on the elements sharing the interface, it follows that the face bubble functions are $\boldsymbol{H}$ (curl)-conforming.

Interior functions: Both types of face function have vanishing tangential components on all faces, and are therefore trivially seen to be conforming.

\subsection{H(div)-Conforming Basis Functions}

A conforming discretisation of the space $\boldsymbol{H}(\mathbf{d i v})$ is characterised by the continuity of the normal components across element interfaces. More precisely, Nedelec [29, Lemma 1] shows that if domains $K$ and $K^{\prime}$ share a common face $\boldsymbol{f}$ with normal $\boldsymbol{n}$, then a smooth vector field $\boldsymbol{u}$ on each domain belongs to $\boldsymbol{H}\left(\operatorname{div} ; K \cup K^{\prime}\right)$ provided that $\boldsymbol{n} \cdot \boldsymbol{u}$ is the same on each side of the face $\boldsymbol{f}$. 


\subsubsection{Reference Element Basis Functions}

The vector-valued polynomial space of order $p$ on the reference element $\hat{\boldsymbol{t}}$ is given by

$$
\hat{\boldsymbol{X}}_{p}^{\operatorname{div}}=\left(\mathbb{P}_{p}(\hat{\boldsymbol{t}})\right)^{3} .
$$

A set of hierarchic basis functions for $\hat{\boldsymbol{X}}_{p}^{\text {div }}$ is given in Table 4.

The edge-based face functions defined in Table 4 associated with each edge $\boldsymbol{e}=[o i]$ of face $\boldsymbol{f}$, are linear combinations of the elementary functions

$$
\hat{\varphi}_{\boldsymbol{e}}^{\boldsymbol{f}}=\hat{\lambda}_{\boldsymbol{f} \backslash \boldsymbol{e}} \hat{\nabla} \hat{\lambda}_{o} \wedge \hat{\nabla} \hat{\lambda}_{i}
$$

where $\boldsymbol{f} \backslash \boldsymbol{e}$ denotes the vertex opposite edge $\boldsymbol{e}$ in face $\boldsymbol{f}$. These functions are used in [7] to construct a basis for the lowest order, or Whitney, elements in the form

$$
2 \sum_{e \subset \partial f} \hat{\varphi}_{e}^{f}
$$

It is not difficult to verify that the normal component of the function $\hat{\varphi}_{e}^{f}$ on a face $\boldsymbol{f}^{\prime}$ vanishes unless $\boldsymbol{f}^{\prime}$ and $\boldsymbol{f}$ coincide,

$$
n^{f^{\prime}} \cdot \hat{\varphi}_{e}^{f}=0, \quad f \neq f^{\prime} .
$$

There are $p$ edge-based face functions associated with each of the three edges of face $\boldsymbol{f}$, giving in total, $12 p$ functions on the tetrahedron.

The normal component of the face bubble functions vanishes on all edges and on all faces except the face with which they are associated. There are $(p-2)(p-1) / 2$ functions per face, and consequently $2(p-2)(p-1)$ face bubble functions altogether on the tetrahedron.

There are three types of interior function. The normal components of all types of interior function vanish on all faces of the tetrahedron. The edgebased interior functions have non-zero tangential components on the edge with which they are associated and account for $6(p-1)$ basis functions in total. The face-based interior functions have vanishing tangential components on all edges, but have non-zero tangential components on the face with which they are associated. These account for $2(p-1)(p-2)$ basis functions. Finally, the interior bubble functions vanish on all boundaries and account for $(p-3)(p-2)(p-1) / 2$ functions in total.

These observations are used to prove the following result:

Lemma 6 The set of functions defined in Table 4 forms a hierarchic basis for the spaces $\hat{\boldsymbol{X}}_{p}^{\mathbf{d i v}}, p \in \mathbb{N}$.

Proof. Each of the functions defined in Table 4 belongs to the space $\left(\mathbb{P}_{p}\right)^{3}$. The next step is to show the functions are linearly independent. Suppose that

$$
\sum_{f} \sum_{e \subset \partial f} w_{e}^{f}+\sum_{f} w^{f}+\sum_{e \in \mathcal{E}} w_{e}^{t}+\sum_{f} w_{f}^{t}+w^{t} \equiv 0
$$

where $\boldsymbol{w}_{e}^{f}, \boldsymbol{w}^{f}, \boldsymbol{w}_{e}^{t}, \boldsymbol{w}_{f}^{t}$ and $\boldsymbol{w}^{t}$ are linear combinations of face and interior functions respectively. The basis functions within these categories are linearly 


$$
\text { Space: }\left(\mathbb{P}_{p}(\hat{\boldsymbol{t}})\right)^{3}, p \in \mathbb{N}
$$

Edge-Based Face Functions: $\boldsymbol{f} \in \mathcal{F}(\hat{\boldsymbol{t}})$

For each edge $\boldsymbol{e}=[o i] \subset \partial \boldsymbol{f}$ :

$$
\hat{\varphi}_{\boldsymbol{e}, \ell}^{f}=\psi_{\ell}\left(\xi_{\boldsymbol{e}}\right) \hat{\varphi}_{e}^{f}, \quad 0 \leq \ell \leq p-1
$$

where

$$
\hat{\varphi}_{e}^{f}=\hat{\lambda}_{\boldsymbol{f} \backslash \boldsymbol{e}} \hat{\nabla} \hat{\lambda}_{o} \wedge \hat{\nabla} \hat{\lambda}_{i}
$$

and $\boldsymbol{f} \backslash \boldsymbol{e}$ denotes the vertex opposite edge $\boldsymbol{e}$ in face $\boldsymbol{f}$.

Face Bubble Functions: $\boldsymbol{f}=[o i j] \in \mathcal{F}(\hat{\boldsymbol{t}})$

$\hat{\boldsymbol{\varphi}}_{\ell m}^{f}=\beta_{o i j} \psi_{\ell}\left(\hat{\xi}_{o i}\right) \psi_{m}\left(\hat{\xi}_{o j}\right) \hat{\nabla} \hat{\lambda}_{i} \wedge \hat{\nabla} \hat{\lambda}_{j}, \quad 0 \leq \ell, m, \ell+m \leq p-3$

Edge-Based Interior Functions: $\boldsymbol{e} \in \mathcal{E}(\hat{\boldsymbol{t}})$

$$
\hat{\boldsymbol{\varphi}}_{\boldsymbol{e}, \ell}^{\hat{\boldsymbol{t}}}=\beta_{\boldsymbol{e}} \psi_{\ell}\left(\hat{\xi}_{o i}\right) \hat{\boldsymbol{\tau}}^{e}, \quad 0 \leq \ell \leq p-2
$$

Face-Based Interior Functions: $\boldsymbol{f}=[o i j] \in \mathcal{F}(\hat{\boldsymbol{t}})$

$$
\left.\begin{array}{l}
\hat{\boldsymbol{\varphi}}_{\boldsymbol{f}, i, \ell m}^{\hat{\boldsymbol{t}}}=\beta_{o i j} \psi_{\ell}\left(\hat{\xi}_{o i}\right) \psi_{m}\left(\hat{\xi}_{o j}\right) \hat{\boldsymbol{\tau}}^{[o i]} \\
\hat{\boldsymbol{\varphi}}_{\boldsymbol{f}, j, \ell m}=\beta_{o i j} \psi_{\ell}\left(\hat{\xi}_{o i}\right) \psi_{m}\left(\hat{\xi}_{o j}\right) \hat{\boldsymbol{\tau}}^{[o j]}
\end{array}\right\} \quad 0 \leq \ell, m, \ell+m \leq p-3
$$

Interior Bubble Functions: $\hat{\boldsymbol{t}}=[o i j k]$

$$
\begin{array}{r}
\hat{\boldsymbol{\varphi}}_{d, \ell m n}^{\hat{\boldsymbol{t}}}=\beta_{\boldsymbol{t}} \psi_{\ell}\left(\hat{\xi}_{o i}\right) \psi_{m}\left(\hat{\xi}_{o j}\right) \psi_{n}\left(\hat{\xi}_{o k}\right) \hat{\boldsymbol{e}}_{d}, \quad d \in\{1,2,3\} \\
0 \leq \ell, m, n, \ell+m+n \leq p-4
\end{array}
$$

Table 4: Hierarchic basis functions for $\boldsymbol{H}(\mathbf{d i v})$-conforming finite element space of order $p$. 
independent, and it therefore suffices to show (19) implies the each of the above functions must vanish individually.

Taking inner products of (19) with $\boldsymbol{n}^{\boldsymbol{f}}$ and evaluating on a face $\boldsymbol{f} \in \mathcal{F}$ gives

$$
\left.\sum_{e \subset \partial f} n^{f} \cdot w_{e}^{f}\right|_{f}+\left.n^{f} \cdot w^{f}\right|_{f}=0
$$

since the normal components of the interior functions vanish on all faces. Recalling that the face bubble functions vanish on all edges and using properties of the edge-based face functions reveals firstly that $\boldsymbol{w}_{\boldsymbol{e}}^{f}=\mathbf{0}$, and hence that $\boldsymbol{w}^{f}=\mathbf{0}$.

Consequently, the first two terms in (19) vanish. The remaining terms vanish too. Firstly, the edge-based interior functions are the only non-zero functions on the edges and must therefore be zero. Then, the face-based interior functions are the only remaining non-zero functions on the faces and must also vanish identically. Thus, equation (19) reduces to $\boldsymbol{w}^{\boldsymbol{t}}=\mathbf{0}$ and it follows that the basis functions are linearly independent.

The proof is concluded by observing that the number of basis functions defined in Table 4 coincides with the dimension, $(p+1)(p+2)(p+3) / 2$, of the space $\left(\mathbb{P}_{p}\right)^{3}$.

\subsubsection{Global Basis Functions}

A global basis function $\boldsymbol{\varphi}$ of $\boldsymbol{H}(\mathbf{d i v})$ defined on a physical element $\boldsymbol{t} \in \mathcal{T}$ is constructed from the basis function $\hat{\varphi}$ defined on the reference element using the contravariant (Piola) transformation [25]:

$$
\left.\varphi\right|_{\boldsymbol{t}}=\frac{1}{\operatorname{det}\left(\boldsymbol{J}_{\boldsymbol{t}}\right)}\left(\boldsymbol{J}_{\boldsymbol{t}} \hat{\boldsymbol{\varphi}}\right) \circ \boldsymbol{F}_{\boldsymbol{t}}^{-1} .
$$

The Piola transformation has the follow important property

$$
\frac{1}{\operatorname{det}\left(\boldsymbol{J}_{\boldsymbol{t}}\right)} \boldsymbol{J}_{\boldsymbol{t}}\left(\hat{\nabla} \lambda_{o} \wedge \hat{\nabla} \lambda_{i}\right)=\nabla \lambda_{o} \wedge \nabla \lambda_{i} .
$$

The global discrete $\boldsymbol{H}(\mathbf{d i v})$ space of order $p$ is defined by

$$
\begin{aligned}
\boldsymbol{X}_{p, \mathcal{T}}^{\mathbf{d i v}}= & \bigoplus_{\boldsymbol{f} \in \mathcal{F}} \operatorname{span}\left\{\boldsymbol{\varphi}_{\boldsymbol{e}, \ell}^{\boldsymbol{f}}: 0 \leq \ell \leq p-1, \boldsymbol{e} \subset \partial \boldsymbol{f}\right\} \\
& \bigoplus_{\boldsymbol{f} \in \mathcal{F}} \operatorname{span}\left\{\boldsymbol{\varphi}_{\ell m}^{\boldsymbol{f}}: 0 \leq \ell, m, \ell+m \leq p-3,\right\} \\
& \bigoplus_{\boldsymbol{t} \in \mathcal{T}} \operatorname{span}\left\{\boldsymbol{\varphi}_{\boldsymbol{e}, \ell}^{\boldsymbol{t}}: 0 \leq \ell \leq p-2, \boldsymbol{e} \subset \partial \boldsymbol{t}\right\} \\
& \bigoplus_{\boldsymbol{t} \in \mathcal{T}} \operatorname{span}\left\{\boldsymbol{\varphi}_{\boldsymbol{f}, i, \ell m}^{\boldsymbol{t}}, \boldsymbol{\varphi}_{\boldsymbol{f}, j, \ell m}^{\boldsymbol{t}}: 0 \leq \ell, m \leq p-3, \boldsymbol{f}=[o i j] \in \mathcal{F}(\boldsymbol{t})\right\} \\
& \bigoplus_{\boldsymbol{t} \in \mathcal{T}} \operatorname{span}\left\{\boldsymbol{\varphi}_{d, \ell m n}^{\boldsymbol{t}}: d \in\{1,2,3\}, 0 \leq \ell, m, n \leq p-4\right\} .
\end{aligned}
$$

This constitutes an $\boldsymbol{H}(\mathbf{d i v})$-conforming basis: 
Theorem $\mathbf{7}$ The space $\boldsymbol{X}_{p, \mathcal{T}}^{\mathbf{d i v}}$ is an $\boldsymbol{H}(\mathbf{d i v})$-conforming subspace of order $p$.

Proof. Fix $p \in \mathbb{N}$. Lemma 6 shows that the space is of $p$-th order and it suffices to show that if distinct elements $\boldsymbol{t}$ and $\boldsymbol{t}^{\prime}$ share a common face $\boldsymbol{f}$, then for each global basis function, the quantity $\boldsymbol{n}^{\boldsymbol{f}} \cdot \boldsymbol{\varphi}$ is continuous across the face $\boldsymbol{f}$.

Interior functions: All types of face function have vanishing normal components on all faces, and are therefore $\boldsymbol{H}(\mathbf{d i v})$-conforming.

Face functions: Let $\boldsymbol{e}=[o i] \subset \partial \boldsymbol{f}$ be an edge of the shared face. The face functions are combinations of functions of $\xi_{e}$ multiplied by the images of products of the form $\hat{\nabla} \hat{\lambda}_{i} \wedge \hat{\nabla} \hat{\lambda}_{j}$ under the Piola transformation, which, in view of relation (21) are given by $\nabla \lambda_{i} \wedge \nabla \lambda_{j}$. The coordinate $\xi_{e}$ is continuous on the shared face, and it suffices to show that the normal component of $\nabla \lambda_{i} \wedge \nabla \lambda_{j}$ is continuous across the face. This quantity only depends on the tangential components of $\nabla \lambda_{o}$ and $\nabla \lambda_{i}$ on $\boldsymbol{f}$. However, the barycentric coordinates are continuous and thus the tangential components of their gradients is continuous across a face and it follows that the normal components of the face functions match across a face.

\section{Implementation}

The application of the basis functions to the situation where elements of nonuniform local polynomial order are required is now considered. The generalisation to non-uniform order is straightforward provided a hierarchical basis is available once it is realised [2], that one only need construct the connectivity mappings.

Connectivity mappings play a key role in finite element analysis. For instance, they are used to distribute the global solution vector to the elements and in the assembly of the global linear system. The standard finite element sub-assembly procedure for a global matrix $\boldsymbol{M}$, such as the mass matrix, may be written in the form $[2,22]$ :

$$
M=\sum_{t \in \mathcal{T}} \Lambda_{t} M_{t} \Lambda_{t}^{\top}
$$

where $\boldsymbol{M}_{\boldsymbol{t}}$ are element matrices and $\boldsymbol{\Lambda}_{\boldsymbol{t}}$ are connectivity mappings.

A connectivity mapping is represented by a rectangular Boolean matrix of dimension $N \times N_{\boldsymbol{t}}$ where $N$ is the total number of global degrees of freedom and $N_{\boldsymbol{t}}$ is the number of degrees of freedom on element $\boldsymbol{t}$. The entries of the matrix are given by

$$
\left[\boldsymbol{\Lambda}_{\boldsymbol{t}}\right]_{i j}= \begin{cases}1 & \text { if } i \text { is the global number of local degree of freedom } j \\ 0 & \text { otherwise. }\end{cases}
$$

To re-iterate, the construction of the connectivity mappings is the only modification of the standard finite element methodology needed to implement locally non-uniform order approximation. All other operations such as the evaluation of the element matrices, application of boundary conditions etc. follow the usual approach. 


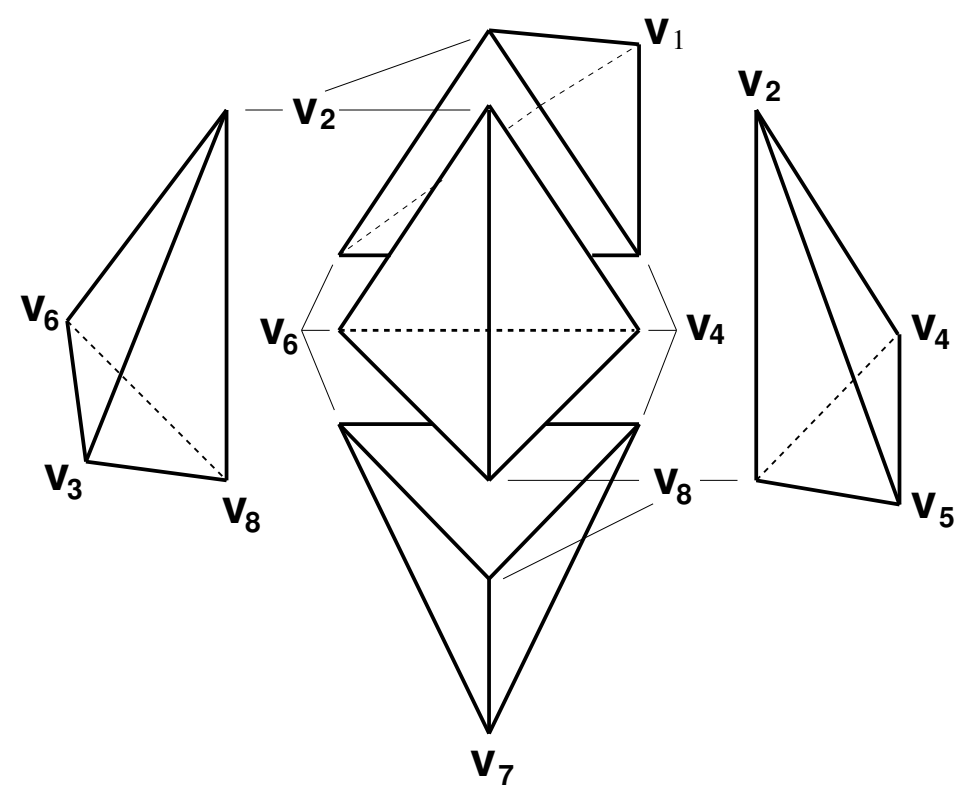

Figure 7: Exploted view of five-element mesh used to illustrate variable order implementation.

The implementation of the bases for non-uniform local polynomial order will be illustrated by considering a simple mesh comprised of five elements

$$
\mathcal{T}=\left\{\boldsymbol{t}_{1}, \ldots, \boldsymbol{t}_{5}\right\} .
$$

shown in Figure 7. The elements and the desired polynomial orders are given by

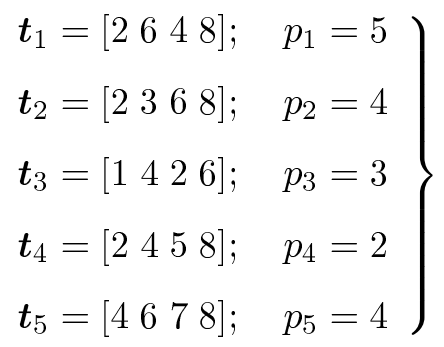

where the elements have been oriented as described in the previous section. The first element $\boldsymbol{t}_{1}$ represents the most common situation whereby an element has neighbouring elements on all faces. Here, in order to avoid a proliferation of data, only the information needed for element $\boldsymbol{t}_{1}$ will be presented in detail, since the treatment of larger number of elements follows the same pattern.

The vertices $\mathcal{V}\left(\boldsymbol{t}_{1}\right)$, edges $\boldsymbol{E}\left(\boldsymbol{t}_{1}\right)$ and faces $\mathcal{F}\left(\boldsymbol{t}_{1}\right)$ of element $\boldsymbol{t}_{1}$ are easily identified from the global vertex numbers [ $\left[\begin{array}{llll}2 & 6 & 4 & 8\end{array}\right]$ of the element:

$$
\begin{aligned}
& \mathcal{V}\left(\boldsymbol{t}_{1}\right)=\{2,4,6,8\} \\
& \mathcal{E}\left(\boldsymbol{t}_{1}\right)=\left\{\boldsymbol{e}_{1}, \boldsymbol{e}_{2}, \ldots, \boldsymbol{e}_{6}\right\} \\
& \mathcal{F}\left(\boldsymbol{t}_{1}\right)=\left\{\boldsymbol{f}_{1}, \boldsymbol{f}_{2}, \boldsymbol{f}_{3}, \boldsymbol{f}_{4}\right\}
\end{aligned}
$$




\begin{tabular}{|c|c|c|c|}
\hline Entity & Vertices & $\begin{array}{c}\text { Contained } \\
\text { Within }\end{array}$ & $\begin{array}{l}\text { Local } \\
\text { Order }\end{array}$ \\
\hline$e_{1}$ & {$\left[\begin{array}{ll}2 & 6\end{array}\right]$} & $\boldsymbol{t}_{1}, \boldsymbol{t}_{2}, \boldsymbol{t}_{3}$ & 3 \\
\hline$e_{2}$ & {$\left[\begin{array}{ll}2 & 8\end{array}\right]$} & $\boldsymbol{t}_{1}, \boldsymbol{t}_{2}, \boldsymbol{t}_{4}$ & 2 \\
\hline$e_{3}$ & {$\left[\begin{array}{ll}6 & 8\end{array}\right]$} & $\boldsymbol{t}_{1}, \boldsymbol{t}_{2}, \boldsymbol{t}_{5}$ & 1 \\
\hline$e_{4}$ & {$\left[\begin{array}{ll}2 & 4\end{array}\right]$} & $t_{1}, t_{3}, t_{4}$ & 2 \\
\hline$e_{5}$ & {$\left[\begin{array}{ll}4 & 6\end{array}\right]$} & $t_{1}, t_{3}, t_{5}$ & 1 \\
\hline$e_{6}$ & {$\left[\begin{array}{ll}4 & 8\end{array}\right]$} & $t_{1}, t_{4}, t_{5}$ & 1 \\
\hline$f_{1}$ & {$\left[\begin{array}{lll}2 & 6 & 8\end{array}\right]$} & $t_{1}, t_{2}$ & 4 \\
\hline$f_{2}$ & {$\left[\begin{array}{lll}2 & 4 & 6\end{array}\right]$} & $t_{1}, t_{3}$ & 3 \\
\hline$f_{3}$ & {$\left[\begin{array}{lll}2 & 4 & 8\end{array}\right]$} & $t_{1}, t_{4}$ & 2 \\
\hline $\boldsymbol{f}_{4}$ & {$\left[\begin{array}{lll}4 & 6 & 8\end{array}\right]$} & $t_{1}, t_{5}$ & 1 \\
\hline
\end{tabular}

Table 5: Edges and faces of element $\boldsymbol{t}_{1}$ and local order of approximation determined from using minimum degree of elements containing the entity.

where the edges and faces are defined in Table 5. The complete sets $\mathcal{V}, \mathcal{E}$ and $\mathcal{F}$ of vertices, edges and interiors are compiled by forming the unions of such elemental contributions by looping over elements. At the same time, the active polynomial order applied on each entity is also be determined by applying a minimum rule [2]. This rule states that the active order of approximation an edge or face is taken to be the minimum degree of all elements containing the edge or face. For instance, face $\boldsymbol{f}_{1}$ is shared by elements $\boldsymbol{t}_{1}$ and $\boldsymbol{t}_{2}$, having local orders 5 and 4 respectively. Applying the minimum rule gives a local order of approximation $\min (4,5)=4$ on face $\boldsymbol{f}_{1}$. The local order of approximation on edge $\boldsymbol{e}_{1}$ is the minimum order of the elements $\boldsymbol{t}_{1}, \boldsymbol{t}_{2}$ and $\boldsymbol{t}_{3}$ containing the edge, giving an order $\min (5,4,3)=3$ on the edge. Table 5 shows the local orders approximation on all edges and faces of element $\boldsymbol{t}_{1}$.

Once all vertices, edges, faces and interiors have been identified, global numbers are assigned to the degrees of freedom and the connectivity mapping is constructed. This process is performed as follows:

1. For each entity $c \in \mathcal{V} \cup \mathcal{E} \cup \mathcal{F} \cup \mathcal{T}$, (i.e. each element vertex, edge, face and interior):

(a) Find the local order of approximation $p_{c}$.

(b) Determine the number of degrees of freedom $N\left(\boldsymbol{c}, p_{\boldsymbol{c}}\right)$ needed on the entity by reference to Table 6 .

(c) Assign global numbers and distribute to all elements containing the entity.

2. For each element $\boldsymbol{t} \in \mathcal{T}$ : Construct the connectivity mapping $\boldsymbol{\Lambda}_{\boldsymbol{t}}$.

The following sections detail the construction of the connectivity mappings for the five-element mesh described above. 


\begin{tabular}{ccccc} 
Space & Vertices & Edges & Faces & Interiors \\
\hline$H^{1}$ & 1 & $p-1$ & $(p-2)(p-1) / 2$ & $(p-3)(p-2)(p-1) / 6$ \\
$\boldsymbol{H}(\mathbf{c u r l})$ & 0 & $p+1$ & $(p-1)(p+1)$ & $(p-2)(p-1)(p+1) / 2$ \\
$\boldsymbol{H}($ div $)$ & 0 & 0 & $(p+1)(p+2) / 2$ & $(p-1)(p+1)(p+2) / 2$ \\
$L_{2}$ & 0 & 0 & 0 & $(p+1)(p+2)(p+3) / 6$ \\
\hline
\end{tabular}

Table 6: Number of degrees of freedom $N(\boldsymbol{c}, p)$ needed on each entity $\boldsymbol{c}$ (i.e. vertex, edge, face or interior) for the various spaces if the active order of approximation on the entity is $p \in \mathbb{N}$. (Negative quantities correspond to no basis functions.)

\section{1 $\quad L_{2}$-Conforming Subspace}

The basis functions for an $L_{2}$-conforming subspace given in Table 1 consist entirely of interior functions and global numbers are needed only for the interior degrees of freedom, as indicated in Table 6. The local order of approximation on element $\boldsymbol{t}_{1}$ is $p=5$ and Table 6 indicates that 56 degrees of freedom are assigned to element $\boldsymbol{t}_{1}$.

Suppose that the global numbers $\{116, \ldots, 171\}$ are assigned. In theory, the connectivity mapping $\boldsymbol{\Lambda}_{1}$ will be a rectangular matrix of size $N \times 56$, where $N$ is the total number of global degrees of freedom with entries defined as in equation (23). In practice, this matrix is never explicitly constructed, and instead the information is stored in the more compact form

$$
\boldsymbol{\Lambda}_{1}=\left[\begin{array}{lll}
116 & \ldots & 171
\end{array}\right] .
$$

The correspondence between the numbers and the particular basis functions is a matter of taste, provided that any scheme is used consistently.

\section{2 $H^{1}$-Conforming Subspace}

The basis functions for an $H^{1}$-conforming subspace given in Table 2 are separated into subsets of basis functions associated with the individual vertices, edges, faces and element interior. The connectivity mapping on element $\boldsymbol{t}$ may be decomposed accordingly, giving

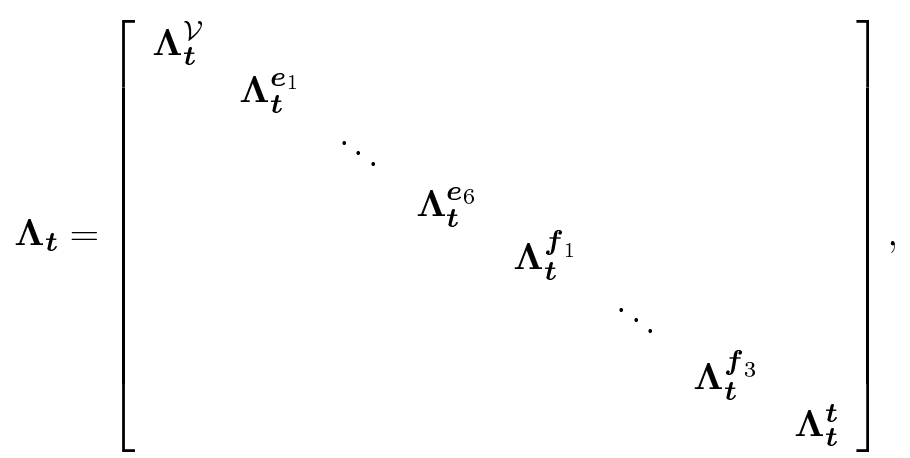


where the remaining entries are zeros, or, more compactly,

$$
\boldsymbol{\Lambda}_{\boldsymbol{t}}=\text { blockdiag }\left[\boldsymbol{\Lambda}_{\boldsymbol{t}}^{\mathcal{V}}, \boldsymbol{\Lambda}_{t}^{e_{1}}, \ldots, \boldsymbol{\Lambda}_{t}^{e_{6}}, \boldsymbol{\Lambda}_{t}^{f_{1}}, \ldots, \boldsymbol{\Lambda}_{t}^{f_{3}}, \boldsymbol{\Lambda}_{t}^{\boldsymbol{t}}\right] .
$$

Table 6 indicates that one degree of freedom per vertex is needed for the space $H^{1}$ giving a total of four for the element. If the actual numbers assigned are selected to coincide with the vertex numbers, then the compact storage scheme described above gives

$$
\boldsymbol{\Lambda}_{1}^{\mathcal{V}}=\left[\begin{array}{llll}
2 & 4 & 6 & 8
\end{array}\right] .
$$

Similarly, Table 5 shows that edge $\boldsymbol{e}_{1}$ has local order of approximation $p=3$ and then Table 6 indicates that two global numbers, $\{9,10\}$ say, are to be assigned. The local order of element $\boldsymbol{t}_{1}$ is $p=5$ meaning that each edge has four basis functions, while only two freedom numbers have been assigned. This is to be expected, since the highest order, $p=4$ and $p=5$, edge functions must have coefficients equal to zero in order to preserve conformity with lower order elements sharing the edge. This is enforced by assigning a global freedom number of ' 0 ' to all such degrees of freedom. The contribution to the connectivity mapping for the element from edge $\boldsymbol{e}_{1}$ is therefore given by

$$
\Lambda_{1}^{e_{1}}=\left[\begin{array}{llll}
9 & 10 & 0 & 0
\end{array}\right] .
$$

Equally well, Table 5 shows that face $\boldsymbol{f}_{1}$ has a local order $p=4$ and then Table 6 indicates that three global numbers, $\{11,12,13\}$ say, are assigned. However, there are six local basis functions on each face of a fifth order element. As described above, the three actual global numbers are padded out with zeros giving

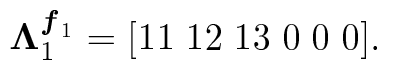

This process continues until finally it is observed that four interior degrees of freedom is needed, $\{85,86,87,88\}$ say, giving

$$
\Lambda_{1}^{\boldsymbol{t}}=\left[\begin{array}{llll}
85 & 86 & 87 & 88
\end{array}\right] .
$$

It is then a simple matter to concatenate the connectivity mappings for each entity to form the element connectivity mapping in the form a string of integers and zeros. The zeros indicate columns of zeros in the full matrix representation and automatically enforce that the coefficients of the associated basis functions must be zero. This is necessary to ensure conformity since these basis functions on the neighbouring, lower order, elements are not activated.

\section{3 $\boldsymbol{H}($ curl $)$ and $\boldsymbol{H}(\operatorname{div})$-Conforming Subspaces}

The basis functions for an $\boldsymbol{H}(\mathbf{c u r l})$-conforming subspace given in Table 3 are separated into subsets of basis functions associated with the edges, faces and element interior. As before, the connectivity mapping on element $\boldsymbol{t}$ may be partitioned into corresponding sub-blocks,

$$
\boldsymbol{\Lambda}_{\boldsymbol{t}}=\text { blockdiag }\left[\boldsymbol{\Lambda}_{\boldsymbol{t}}^{\boldsymbol{e}_{1}}, \ldots, \boldsymbol{\Lambda}_{\boldsymbol{t}}^{\boldsymbol{e}_{6}}, \boldsymbol{\Lambda}_{\boldsymbol{t}}^{\boldsymbol{f}_{1}}, \ldots, \boldsymbol{\Lambda}_{\boldsymbol{t}}^{\boldsymbol{f}_{3}}, \boldsymbol{\Lambda}_{\boldsymbol{t}}^{\boldsymbol{t}}\right],
$$


The contributions from each entity are then constructed using the same procedure as for the space $H^{1}$, with the only difference being that the second row of Table 6 is used to determine the number of degrees of freedom to be assigned.

In the same vein, the basis functions for an $\boldsymbol{H}(\mathbf{d i v})$-conforming subspace given in Table 4 are separated into subsets of basis functions associated with the faces and element interior and the connectivity mapping takes the form

$$
\boldsymbol{\Lambda}_{\boldsymbol{t}}=\text { blockdiag }\left[\boldsymbol{\Lambda}_{\boldsymbol{t}}^{\boldsymbol{f}_{1}}, \ldots, \boldsymbol{\Lambda}_{\boldsymbol{t}}^{\boldsymbol{f}_{3}}, \boldsymbol{\Lambda}_{\boldsymbol{t}}^{\boldsymbol{t}}\right] .
$$

The treatment is then identical with that for the $H^{1}$-case, using the third row of Table 6 to determine the number of degrees of freedom.

It will be noticed that in passing from $H^{1}$ to $\boldsymbol{H}(\mathbf{c u r l})$ to $\boldsymbol{H}(\mathbf{d i v})$, the entity with the lowest dimension is dropped at each step. One might wonder what happens in the next step whereby faces would be removed, yielding a mapping of the form

$$
\boldsymbol{\Lambda}_{\boldsymbol{t}}=\text { blockdiag }\left[\boldsymbol{\Lambda}_{\boldsymbol{t}}^{\boldsymbol{t}}\right] .
$$

This simply corresponds to the $L_{2}$ space considered earlier.

\section{References}

[1] M. Ainsworth and J. Coyle. Hierarchic hp-edge element families for Maxwell's equations on hybrid quadrilateral/triangular meshes. Comput. Methods Appl. Mech. Engrg., 190:6709-6733, 2001.

[2] M. Ainsworth and B. Senior. Aspects of an adaptive $h p$-finite element method: Adaptive strategy, conforming approximation and efficient solvers. Comput. Methods Appl. Mech. Engrg., 150:65-87, 1997.

[3] M. Ainsworth and B. Senior. hp-Finite element procedures on non-uniform geometric meshes: Adaptivity and constrained approximation. In M. W. Bern, J. E. Flaherty, and M. Luskin, editors, Grid Generation and Adaptive Algorithms, volume 113, pages 1-29. IMA, Minnesota, 1999.

[4] J.E. Akin. Finite elements for Analysis and Design. Academic Press, 1994.

[5] L. Andersen and J. Volakis. Hierarchical tangential vector finite elements for tetrahedra. IEEE Microwave and Guided Wave Letters, 8(3):127-129, 1998.

[6] A. Bossavit. A rationale for edge-elements in 3-d fields computations. IEEE Trans. on Magnetics, 24(1):74-79, 1988.

[7] A. Bossavit. Computational Electromagnetism: Variational formulation, complementarity, edge elements. Academic Press, 1998.

[8] A. Bossavit and I. Mayergoyz. Edge elements in scattering problems. IEEE Trans. on Magnetics, 25(4):2816-2821, 1989.

[9] F. Brezzi and M. Fortin. Mixed and hybrid finite element methods. Springer-Verlag, Berlin, 1991. 
[10] Z. Cendes. Vector finite elements for electromagnetic field computation. IEEE Trans. on Magnetics, 27(5):3958-3967, 1991.

[11] P. G. Ciarlet. The Finite Element Method for Elliptic Problems. Elsevier, North-Holland, 1978.

[12] L. Demkowicz, P. Monk, L. Vardapetyan, and W. Rachowicz. De Rham diagram for hp finite element spaces. Technical report, TICAM Report 99-6, University of Texas at Austin, 1999.

[13] L Demkowicz and L Vardapetyan. Modeling of electromagnetic absorption/scattering problems using hp- adaptive finite elements. Comput. Methods Appl. Mech. Engrg., 152(1-2):103-124, 1998.

[14] C. Geuzaine, B. Meys, P. Dular, and W. Legros. Convergence of high order curl-conforming finite elements. IEEE Trans. on Magnetics, 35(3):14421444, 1999.

[15] I.S. Gradshteyn and I.M. Ryzhik. Table of Integrals, Series and Products. Academic Press Ltd, A. Jeffrey (ed.) United Kingdom 5th edition, 1994.

[16] R.D. Graglia, D.R. Wilton, and A.F. Peterson. Higher order interpolatory vector bases for computational electromagnetics. IEEE Trans. on Antennas and Propagation, 45(3):329-342, 1997.

[17] R. Hiptmair. Canonical construction of finite elements. Math. Comp., 68(228):1325-1346, 1999.

[18] J. Jin. The Finite Element Method in Electromagnetics. John Wiley \& Sons, Inc, 1993.

[19] A. Kameari. Calculation of transient 3D eddy current using edge-elements. IEEE Trans. on Magnetics, 26:466-469, 1990.

[20] A. Kameari. Symmetric second order edge elements for triangles and tetrahedra. IEEE Trans. on Magnetics, 35(3):1394-1397, 1999.

[21] G. Karniadakis and S. Sherwin. Spectral/hp element methods for CFD. Oxford University Press, 1999.

[22] G. Kron. A set of principles to interconnect the solutions of physical systems. Journal of Applied Physics, 24(8):965-980, 1953.

[23] J-F. Lee, D-K. Sun, and Z. Cendes. Full-wave analysis of dielectric waveguides using tangential vector finite elements. IEEE Trans. Microwave Theory and Techniques, 39(8):1262-1271, 1991.

[24] J-F. Lee, D-K. Sun, and Z. Cendes. Tangential vector finite elements for electromagnetic field computation. IEEE Trans. on Magnetics, 27(5):40324035, 1991.

[25] J.E. Marsden and T.J.R. Hughes. Mathematical Foundations of Elasticity. Prentice-Hall, 1983. 
[26] G. Mur. Edge elements, their advantages and their disadvantages. IEEE Trans. on Magnetics, 30(5):3552-3557, 1994.

[27] G. Mur and A. de Hoop. A finite element method for three-dimensional electromagnetic fields in inhomogeneous media. IEEE Trans. on Magnetics, 21(6):2188-2191, 1985.

[28] J. Nédélec. Mixed finite elements in $\mathbb{R}^{3}$. Numer. Math., 93:315-341, 1980.

[29] J. Nédélec. A new family of mixed finite elements in $\mathbb{R}^{3}$. Numer. Math., 50:57-81, 1986.

[30] C. Schwab. p- and hp-Finite Element Methods: Theory and Applications in Solid and Fluid Mechanics. Numerical Mathematics and Scientific Computation. Oxford University Press, 1998.

[31] P. Silvester and R. Ferrari. Finite elements for electrical engineers. Cambridge University Press, 1996.

[32] B. Szabo and I. Babuska. Finite Element Analysis. John Wiley \& Sons, 1991.

[33] J.P. Webb. Edge elements and what they can do for you. IEEE Trans. on Antennas and Propagation, 29:1460-1465, 1993.

[34] J.P. Webb. Hierarchal vector basis functions of arbitrary order for triangular and tetrahedral finite elements. IEEE Trans. on Antennas and Propagation, 47(8):1244-1253, AUG 1999.

[35] J.P. Webb and B. Forghani. Hierarchical scalar and vector tetrahedra. IEEE Trans. on Magnetics, 29(2):1495-1498, MAR 1993.

[36] H. Whitney. Geometric Integration Theory. Princeton University Press, 1957. 